This item was submitted to Loughborough's Research Repository by the author.

Items in Figshare are protected by copyright, with all rights reserved, unless otherwise indicated.

\title{
Coupled Ostrovsky equations for internal waves in a shear flow
}

PLEASE CITE THE PUBLISHED VERSION

http://dx.doi.org/10.1063/1.4903279

\section{PUBLISHER}

(c) American Institute of Physics

\section{VERSION}

VoR (Version of Record)

\section{PUBLISHER STATEMENT}

This work is made available according to the conditions of the Creative Commons Attribution-NonCommercialNoDerivatives 4.0 International (CC BY-NC-ND 4.0) licence. Full details of this licence are available at: https://creativecommons.org/licenses/by-nc-nd/4.0/

\section{LICENCE}

CC BY-NC-ND 4.0

\section{REPOSITORY RECORD}

Alias, A., Roger H.J. Grimshaw, and Karima R. Khusnutdinova. 2019. "Coupled Ostrovsky Equations for Internal Waves in a Shear Flow”. figshare. https://hdl.handle.net/2134/20161. 


\section{AIP $\mid$ Physics of Fluids}

\section{Coupled Ostrovsky equations for internal waves in a shear flow}

A. Alias, R. H. J. Grimshaw, and K. R. Khusnutdinova

Citation: Physics of Fluids 26, 126603 (2014); doi: 10.1063/1.4903279

View online: http://dx.doi.org/10.1063/1.4903279

View Table of Contents: http://scitation.aip.org/content/aip/journal/pof2/26/12?ver=pdfcov

Published by the AIP Publishing

\section{Articles you may be interested in}

Vortical disturbances in a linearly stratified linear shear flow. II. Nonlinear evolution

Phys. Fluids 27, 024104 (2015); 10.1063/1.4907187

The mean flow and long waves induced by two-dimensional internal gravity wavepackets

Phys. Fluids 26, 106601 (2014); 10.1063/1.4899262

Transcritical generation of nonlinear internal waves in the presence of background shear flow Phys. Fluids 26, 086601 (2014); 10.1063/1.4891871

Two-dimensional moist stratified turbulence and the emergence of vertically sheared horizontal flows

Phys. Fluids 24, 036602 (2012); 10.1063/1.3694805

Resonant three-wave interaction of Holmboe waves in a sharply stratified shear flow with an inflection-free velocity profile

Phys. Fluids 23, 114101 (2011); 10.1063/1.3657093

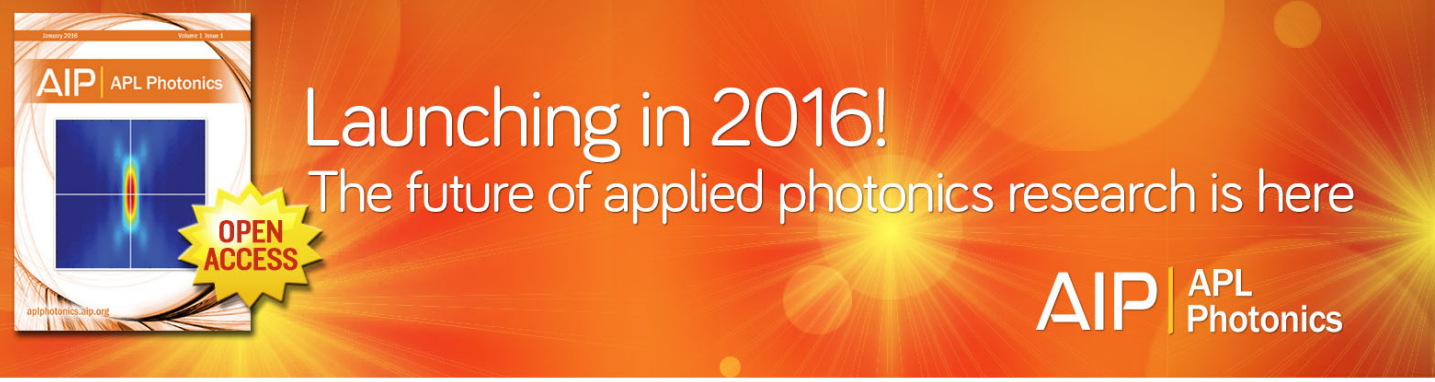




\title{
Coupled Ostrovsky equations for internal waves in a shear flow
}

\author{
A. Alias, ${ }^{1,2}$ R. H. J. Grimshaw ${ }^{2}$ and K. R. Khusnutdinova ${ }^{2, a)}$ \\ ${ }^{1}$ School of Informatics and Applied Mathematics, Universiti Malaysia Terengganu, \\ 21030 Terengganu, Malaysia \\ ${ }^{2}$ Department of Mathematical Sciences, Loughborough University, \\ Loughborough LE11 3TU, United Kingdom
}

(Received 23 May 2014; accepted 4 November 2014; published online 17 December 2014)

\begin{abstract}
In the context of fluid flows, the coupled Ostrovsky equations arise when two distinct linear long wave modes have nearly coincident phase speeds in the presence of background rotation. In this paper, nonlinear waves in a stratified fluid in the presence of shear flow are investigated both analytically, using techniques from asymptotic perturbation theory, and through numerical simulations. The dispersion relation of the system, based on a three-layer model of a stratified shear flow, reveals various dynamical behaviours, including the existence of unsteady and steady envelope wave packets. ( 2014 AIP Publishing LLC. [http://dx.doi.org/10.1063/1.4903279]
\end{abstract}

\section{INTRODUCTION}

It is widely known that the Korteweg-de Vries (KdV) equation, with various extensions, is a canonical model for the description of the nonlinear internal waves that are commonly observed in the oceans, see the reviews Grimshaw, ${ }^{1}$ Grimshaw et al. ${ }^{2}$ Helfrich and Melville, ${ }^{3}$ and references therein. The KdV equation is developed for weakly nonlinear long waves, and importantly in the context of this paper, is derived on the assumption that the dynamics is dominated by a single linear long wave mode. When background rotation is included, the KdV equation is replaced by the Ostrovsky equation, see Ostrovsky, ${ }^{4}$ Leonov, ${ }^{5}$ Helfrich, ${ }^{6}$ Grimshaw, ${ }^{7,8}$ given by, in a reference frame moving with the linear long wave phase speed

$$
\left\{A_{t}+v A A_{x}+\lambda A_{x x x}\right\}_{x}=\gamma A,
$$

where $\gamma$ is the rotation coefficient, and $v$ and $\lambda$ are the nonlinearity and dispersion coefficients, respectively. Here, $A(x, t)$ is the amplitude of the linear long wave mode $\phi(z)$ corresponding to the linear long wave phase speed $c$, which is determined from the modal equations

$$
\begin{aligned}
& \left(\rho_{0} W^{2} \phi_{z}\right)_{z}+\rho_{0} N^{2} \phi=0, \\
& \phi=0 \quad \text { at } z=-h, \text { and } W^{2} \phi_{z}=g \phi \text { at } z=0 .
\end{aligned}
$$

Here, $\rho_{0}(z)$ is the stable background density stratification, $\rho_{0} N^{2}=-g \rho_{0 z}, W=c-u_{0}$ where $u_{0}(z)$ is the background shear flow, and it is assumed that there are no critical levels, that is, $W \neq 0$ for any $z$ in the flow domain. The coefficients are given by

$$
I v=3 \int_{-h}^{0} \rho_{0} W^{2} \phi_{z}^{3} d z, \quad I \lambda=\int_{-h}^{0} \rho_{0} W^{2} \phi^{2} d z, \quad I \gamma=f^{2} \int_{-h}^{0} \rho_{0} \Phi \phi_{z} d z,
$$

where

$$
I=2 \int_{-h}^{0} \rho_{0} W \phi_{z}^{2} d z, \quad \rho_{0} W \Phi=\rho_{0} W \phi_{z}-\left(\rho_{0} u_{0}\right)_{z} \phi
$$

\footnotetext{
a) Author to whom correspondence should be addressed. Electronic mail: K.Khusnutdinova@lboro.ac.uk
} 

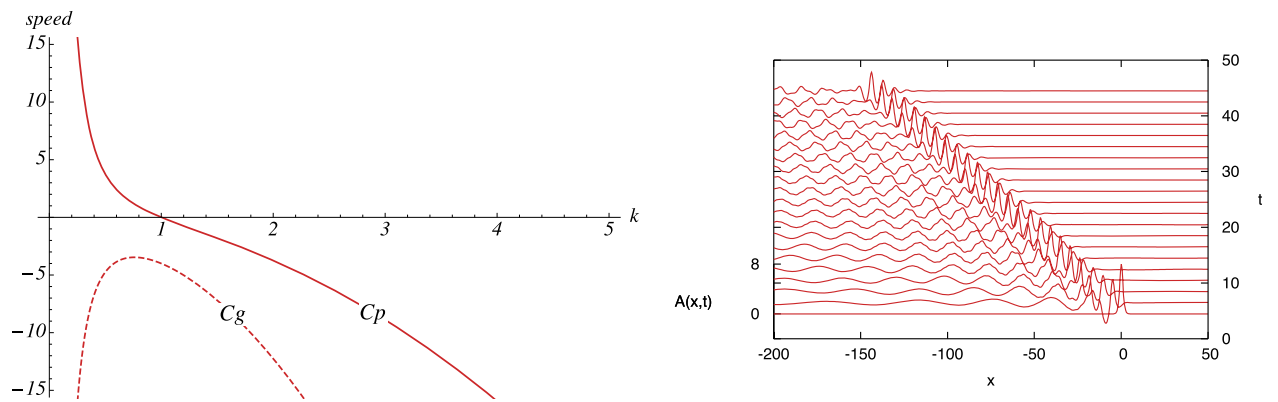

FIG. 1. (Left): Dispersion relation for the Ostrovsky equation (1) when $\lambda \gamma>0$ with $\lambda=\gamma=1$. (Right): Numerical solution of the Ostrovsky equation for an initial condition given by a KdV solitary wave with amplitude 8 at $x=0$.

and $f$ is the Coriolis parameter. Note that when there is no shear flow, that is, $u_{0}(z) \equiv 0$, then $\Phi \equiv \phi_{z}$ and $\gamma=f^{2} / 2 c$; in this case $\lambda \gamma>0$.

The effect of the Earth's rotation for the time evolution of an internal wave becomes important when the wave propagates for several inertial periods. For oceanic internal waves, in the absence of a shear flow, $\lambda \gamma>0$, and then it is known that there are no steady solitary wave solutions of equation (1), see Grimshaw and Helfrich ${ }^{9}$ and the references therein. Recently, it was established that the long-time effect of rotation in this case is the destruction of the initial internal solitary wave by the radiation of small-amplitude inertia-gravity waves, and the emergence of a propagating unsteady nonlinear wave packet, associated with the extremum of the group speed, see Helfrich, ${ }^{6}$ Grimshaw and Helfrich, ${ }^{9}$ Grimshaw et al.,${ }^{10}$ and Grimshaw and Helfrich. ${ }^{11}$ The same phenomenon was observed independently by Yagi and Kawahara ${ }^{12}$ in the context of waves in solids. Indeed, the discrete model in Yagi and Kawahara ${ }^{12}$ can be related to a two-directional generalisation of the Ostrovsky equation derived in Gerkema. ${ }^{13}$ A typical linear dispersion curve and numerical simulation are shown in Figure 1. On the other hand, when $\lambda \gamma<0$ the Ostrovsky equation (1) can support steady envelope wave packets, associated with an extremum of the phase speed, see Galkin and Stepanyants ${ }^{14}$ and Obregon and Stepanyants. ${ }^{15}$ Here, a typical case is shown in Figure 2. We note that Obregon and Stepanyants ${ }^{15}$ derived this case for magneto-acoustic waves in a rotating plasma. Although, this case is not relevant to the ocean in the absence of current shear; as a by-product of the analysis presented here, we will show that sufficiently strong shear near a pycnocline may lead to situations where $\lambda \gamma<0$.

It is known that for internal waves, it is possible for the phase speeds of different modes to be nearly coincident, and then there will be a resonant transfer of energy between the waves, see Eckart. ${ }^{16}$ In this case, the KdV equation is replaced by two coupled $\mathrm{KdV}$ equations, describing a strong interaction between internal solitary waves of different modes, see Gear and Grimshaw, ${ }^{17}$ and Grimshaw. ${ }^{18}$ Various families of solitary waves are supported by coupled KdV equations depending on the structure of the linear dispersion relation: pure solitary waves, generalised solitary waves, and
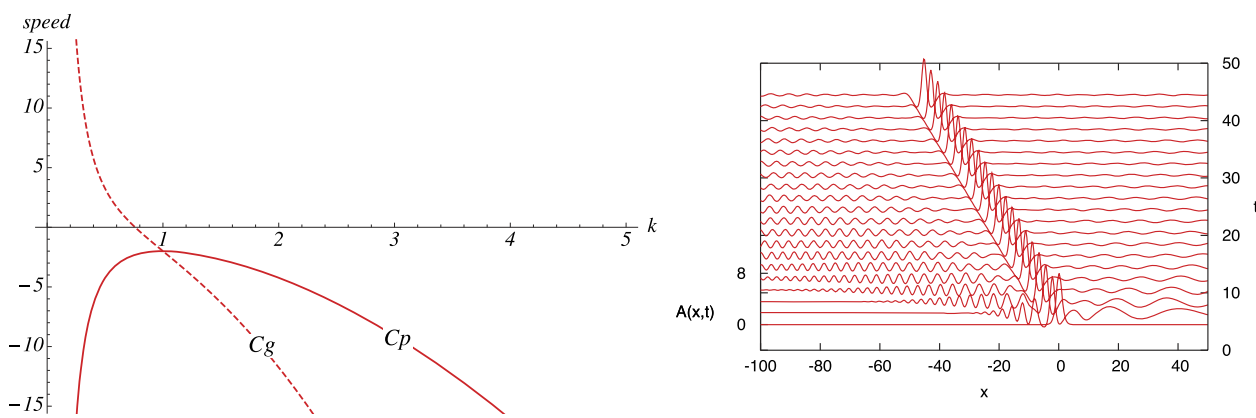

FIG. 2. (Left): Dispersion relation for the Ostrovsky equation (1) when $\lambda \gamma<0$ with $\lambda=-\gamma=1$. (Right): Numerical solution of the Ostrovsky equation for an initial condition given by a KdV solitary wave with amplitude 8 at $x=0$. 
envelope solitary waves, see the review Grimshaw. ${ }^{18}$ In Alias et al. ${ }^{19}$ we extended the derivation of the coupled $\mathrm{KdV}$ equations to take account of background rotation and also a background shear flow. We found that then the single Ostrovsky equation (1) is replaced by two coupled Ostrovsky equations, each equation having both linear and nonlinear coupling terms, given by

$$
\begin{aligned}
& I_{1}\left(A_{1 \tau}+\mu_{1} A_{1} A_{1 s}+\lambda_{1} A_{1 s s s}-\gamma_{1} B_{1}\right) \\
& \quad+v_{1}\left[A_{1} A_{2}\right]_{s}+v_{2} A_{2} A_{2 s}+\lambda_{12} A_{2 s s s}-\gamma_{12} B_{2}=0, \\
& I_{2}\left(A_{2 \tau}+\mu_{2} A_{2} A_{2 s}+\lambda_{2} A_{2 s s s}+\Delta A_{2 s}-\gamma_{2} B_{2}\right) \\
& +v_{2}\left[A_{1} A_{2}\right]_{s}+v_{1} A_{1} A_{1 s}+\lambda_{21} A_{1 s s s}-\gamma_{21} B_{1}=0,
\end{aligned}
$$

where $B_{1 s}=A_{1}, B_{2 s}=A_{2}$. The derivation of (6) and (7) from the fully nonlinear Euler equations is briefly described in Subsection II A and detailly in Alias et al. ${ }^{19}$ Coupled Ostrovsky equations also arise in the context of waves in layered elastic waveguides, see Khusnutdinova et al. ${ }^{20}$ and Khusnutdinova and Moore. ${ }^{21}$ Thus, this model belongs to the class of canonical mathematical models for nonlinear waves, inviting a detailed study of the dynamics of its solutions.

In our previous paper, ${ }^{19}$ we examined in detail the case when there is no background shear flow, and then the coefficients $\gamma, v$ vanish and $\beta=\mu$, leading to a simplification of the underlying linear dispersion relation. In this paper, we restore the background shear flow and find that the range of dynamical behaviours is then greatly extended. The rest of the paper is organised as follows. In Sec. II A, we briefly overview the derivation of a pair of coupled Ostrovsky equations from the complete set of equations of motion for an inviscid, incompressible, density stratified fluid with boundary conditions appropriate to an oceanic situation, using the asymptotic multiple-scales expansions. The effect of background shear is examined using a three-layer model in Sec. II B. In Sec. III, we analyse various cases for the linear dispersion relation. In Sec. IV, based on the analysis of the linear dispersion relation, we present some numerical simulations using a pseudo-spectral method. Some conclusions are drawn in Sec. V.

Our results show that a background shear flow allows for configurations when initial KdV solitary-like waves in the coupled system are destroyed and replaced by a variety of nonlinear envelope wave packets. Two principal types are found; first there are unsteady envelope wave packets, which constitute a two-component counterpart of the outcome for the single Ostrovsky equation (1) with $\lambda \gamma>0$ and are associated with an extremum for the group velocity; second, there are steady wave packets, which are not found for the single Ostrovsky equation with $\lambda \gamma>0$, are associated with an extremum in the phase velocity, and constitute a two-component counterpart of the outcome for the single Ostrovsky equation (1) when $\lambda \gamma<0$. Overall, the dynamics of solutions of the coupled equations is much more complicated. However, the main features of the complex dynamics observed in numerical simulations can be classified and explained in terms of the behaviour of the relevant dispersion curves.

\section{COUPLED OSTROVSKY EQUATIONS}

\section{A. Derivation}

We consider the two-dimensional flow of an inviscid, incompressible fluid on an $f$-plane. In the basic state, the fluid has a density stratification $\rho_{0}(z)$, a corresponding pressure $p_{0}(z)$ such that $p_{0 z}=-g \rho_{0}$, and a horizontal shear flow $u_{0}(z)$ in the $x$-direction. When $u_{0} \neq 0$, this basic state is maintained by a body force. Then, the equations of motion relative to this basic state are given by

$$
\begin{aligned}
\rho_{0}\left(u_{t}+u_{0} u_{x}+w u_{0 z}\right)+p_{x} & =-\left(\rho_{0}+\rho\right)\left(u u_{x}+w u_{z}-f v\right)-\rho\left(u_{t}+u_{0} u_{x}+w u_{0 z}\right), \\
\rho_{0}\left(v_{t}+u_{0} v_{x}+f u\right)+\rho f u_{0} & =-\left(\rho_{0}+\rho\right)\left(u v_{x}+w v_{z}\right)-\rho\left(v_{t}+u_{0} v_{x}\right)-\rho f u \\
p_{z}+g \rho & =-\left(\rho_{0}+\rho\right)\left(w_{t}+\left(u_{0}+u\right) w_{x}+w w_{z}\right) \\
g\left(\rho_{t}+u_{0} \rho_{x}\right)-\rho_{0} N^{2} w & =-g\left(u \rho_{x}+w \rho_{z}\right) \\
u_{x}+w_{z} & =0
\end{aligned}
$$


Here, the terms $\left(u_{0}+u, v, w\right)$ are the velocity components in the $(x, y, z)$ directions, $\rho_{0}+\rho$ is the density, $p_{0}+p$ is the pressure, $t$ is the time, $N(z)$ is the buoyancy frequency, defined by $\rho_{0} N^{2}=-g \rho_{0 z}$, and $f$ is the Coriolis frequency. The free surface and rigid bottom boundary conditions to the above problem are given by

$$
\begin{aligned}
& p_{0}+p=0 \quad \text { at } \quad z=\eta, \\
& \eta_{t}+\left(u_{0}+u\right) \eta_{x}=w \quad \text { at } \quad z=\eta, \\
& w=0 \quad \text { at } \quad z=-h .
\end{aligned}
$$

The constant $h$ denotes the undisturbed depth of the fluid and the function $\eta$ denotes the displacement of the free surface from its undisturbed position $z=0$. A new variable $\zeta$ denotes the vertical particle displacement, which is related to the vertical speed, $w$. It is defined by the equation

$$
\zeta_{t}+\left(u_{0}+u\right) \zeta_{x}+w \zeta_{z}=w
$$

and satisfies the boundary condition

$$
\zeta=\eta \quad \text { at } \quad z=\eta
$$

The system of coupled Ostrovsky equations is derived using the Eulerian formulation, following a similar strategy to the derivation of coupled $\mathrm{KdV}$ equations using the Lagrangian formulation in Gear and Grimshaw, ${ }^{17}$ and Grimshaw; ${ }^{18}$ the full derivation can be found in Alias et al. ${ }^{19}$ At the leading linear long wave order, and in the absence of any rotation, the solution for $\zeta$ is given by an expression of the form $A(x-c t) \phi(z)$ where the modal function is given by (2) and (3). In general, there is an infinite set of solutions for $[\phi(z), c]$. Here, we consider the case when there are two modes with nearly coincident speeds $c_{1}=c$ and $c_{2}=c+\epsilon^{2} \Delta, \epsilon \ll 1$, where $\Delta$ is the detuning parameter. Importantly, we assume that the modal functions $\phi_{1}(z), \phi_{2}(z)$ are distinct, and each satisfy the system (2) and (3), that is,

$$
\begin{aligned}
& \left(\rho_{0} W_{i}^{2} \phi_{i z}\right)_{z}+\rho_{0} N^{2} \phi_{i}=0, \quad i=1,2 \\
& \phi_{i}=0 \quad \text { at } \quad z=-h, \quad \text { and } W_{i}^{2} \phi_{i z}=g \phi_{i} \quad \text { at } \quad z=0 .
\end{aligned}
$$

Here, $W_{i}=c_{i}-u_{0}(z)$ where $c_{i}$ is the long wave speed corresponding to the mode $\phi_{i}(z), i=1,2$. In the sequel, $W_{i}=W=c-u_{0}(z)$ with an error of order $\epsilon^{2}$.

Next, we introduce the scaled variables

$$
\tau=\epsilon \alpha t, \quad s=\epsilon(x-c t), \quad f=\alpha \tilde{f},
$$

where $\alpha=\epsilon^{2}$ and seek a solution in the form of asymptotic multiple-scales expansions

$$
\begin{aligned}
(\zeta, u, \rho, p) & =\alpha\left(\zeta_{1}, u_{1}, \rho_{1}, p_{1}\right)+\alpha^{2}\left(\zeta_{2}, u_{2}, \rho_{2}, p_{2}\right)+\cdots, \\
(w, v) & =\alpha \epsilon\left(w_{1}, v_{1}\right)+\alpha^{2} \epsilon\left(w_{2}, v_{2}\right)+\cdots .
\end{aligned}
$$

Substituting these expansions into the system (8)-(12) and assuming that two waves $A_{1}$ and $A_{2}$ are present at the leading order, we obtain

$$
\begin{aligned}
\zeta_{1} & =A_{1}(s, \tau) \phi_{1}(z)+A_{2}(s, \tau) \phi_{2}(z), \\
u_{1} & =A_{1}\left\{W \phi_{1}\right\}_{z}+A_{2}\left\{W \phi_{2}\right\}_{z}, \\
w_{1} & =-A_{1 s} W \phi_{1}-A_{2 s} W \phi_{2}, \\
p_{1} & =\rho_{0} A_{1} W^{2} \phi_{1 z}+\rho_{0} A_{2} W^{2} \phi_{2 z}, \\
g \rho_{1} & =\rho_{0} N^{2} \zeta_{1}, \\
v_{1} & =\tilde{f}\left(B_{1} \Phi_{1}+B_{2} \Phi_{2}\right), \quad \rho_{0} W \Phi_{1,2}=\rho_{0} W \phi_{1 z, 2 z}-\left(\rho_{0} u_{0}\right)_{z} \phi_{1,2}, \quad B_{1 s, 2 s}=A_{1,2} .
\end{aligned}
$$

Importantly, the exact solution of the linearised equations should contain the exact expressions $W_{1}$ and $W_{2}$ in the terms related to the first and second waves, respectively, rather than just $W$. This difference between the exact and leading order solutions necessitates the introduction of correction terms at the next order, in order to recover the distinct modal equations for the functions $\phi_{1}$ and $\phi_{2}$. 
Collecting terms of the second order for each equation and calculating the correction terms originating from the leading order, the following equations are obtained:

$$
\begin{aligned}
\rho_{0}\left(-W u_{2 s}+u_{0 z} w_{2}\right)+p_{2 s} & =-\rho_{0}\left(u_{1 \tau}+u_{1} u_{1 s}+w_{1} u_{1 z}\right)+\rho_{1}\left(W u_{1 s}-u_{0 z} w_{1}\right)+\rho_{0} \tilde{f} v_{1}, \\
\rho_{0}\left(\tilde{f} u_{2}-W v_{2 s}\right)+\rho_{2} \tilde{f} u_{0} & =-\rho_{0}\left(v_{1 \tau}+u_{1} v_{1 s}+w_{1} v_{1 z}\right)+\rho_{1} W v_{1 s}-\rho_{1} \tilde{f} u_{1}, \\
p_{2 z}+g \rho_{2} & =\rho_{0} W w_{1 s}+2 \Delta A_{2}\left\{\rho_{0} W \phi_{2 z}\right\}_{z}, \\
-g W \rho_{2 s}-\rho_{0} N^{2} w_{2} & =-g\left(\rho_{1 \tau}+u_{1} \rho_{1 s}+w_{1} \rho_{1 z}\right), \\
u_{2 s}+w_{2 z} & =0, \\
W \zeta_{2 s}+w_{2} & =\zeta_{1 \tau}+u_{1} \zeta_{1 s}+w_{1} \zeta_{1 z} .
\end{aligned}
$$

Similarly, the boundary conditions (15), (14), and (17) yield

$$
\begin{aligned}
& w_{2}=0 \quad \text { at } \quad z=-h, \\
& p_{2}-\rho_{0} g \eta_{2}+p_{1 z} \eta_{1}-\frac{1}{2} \rho_{0 z} g \eta_{1}^{2}-2 \Delta \rho_{0} W \phi_{2 z} A_{2}=0 \quad \text { at } \quad z=0, \\
& w_{2}+w_{1 z} \eta_{1}-\eta_{1 \tau}+W \eta_{2 s}-u_{0 z} \eta_{1} \eta_{1 s}-u_{1} \eta_{1 s}=0 \quad \text { at } \quad z=0, \\
& \zeta_{2}+\zeta_{1 z} \eta_{1}-\eta_{2}=0 \quad \text { at } z=0 .
\end{aligned}
$$

Eliminating all variables in favour of $\zeta_{2}$ yields

$$
\begin{aligned}
& \left\{\rho_{0} W^{2} \zeta_{2 s z}\right\}_{z}+\rho_{0} N^{2} \zeta_{2 s}=M_{2} \quad \text { at }-h<z<0, \\
& \zeta_{2}=0 \quad \text { at } \quad z=-h, \quad \rho_{0} W^{2} \zeta_{2 s z}-\rho_{0} g \zeta_{2 s}=N_{2} \text { at } z=0,
\end{aligned}
$$

where $M_{2}, N_{2}$ are known expressions containing terms in $A_{i}$ and their derivatives. The full expressions can be found in Alias et al. ${ }^{19}$

Two compatibility conditions need to be imposed on the system (39) and (40), given by

$$
\int_{-h}^{0} M_{2} \phi_{1,2} d z-\left[N_{2} \phi_{1,2}\right]_{z=0}=0,
$$

where $\phi_{1,2}$ are evaluated at the leading order. These compatibility conditions lead to the coupled Ostrovsky equations

$$
\begin{aligned}
& I_{1}\left(A_{1 \tau}+\mu_{1} A_{1} A_{1 s}+\lambda_{1} A_{1 s s s}-\gamma_{1} B_{1}\right) \\
& +v_{1}\left[A_{1} A_{2}\right]_{s}+v_{2} A_{2} A_{2 s}+\lambda_{12} A_{2 s s s}-\gamma_{12} B_{2}=0, \\
& I_{2}\left(A_{2 \tau}+\mu_{2} A_{2} A_{2 s}+\lambda_{2} A_{2 s s s}+\Delta A_{2 s}-\gamma_{2} B_{2}\right) \\
& +v_{2}\left[A_{1} A_{2}\right]_{s}+v_{1} A_{1} A_{1 s}+\lambda_{21} A_{1 s s s}-\gamma_{21} B_{1}=0,
\end{aligned}
$$

where $B_{1 s}=A_{1}, B_{2 s}=A_{2}$, and the coefficients are given by

$$
\begin{array}{rlrl}
I_{i} \mu_{i} & =3 \int_{-h}^{0} \rho_{0} W^{2} \phi_{i_{z}}^{3} d z, & I_{i} \lambda_{i}=\int_{-h}^{0} \rho_{0} W^{2} \phi_{i}{ }^{2} d z, \\
I_{i} & =2 \int_{-h}^{0} \rho_{0} W \phi_{i_{z}}^{2} d z, & \lambda_{12} & =\lambda_{21}=\int_{-h}^{0} \rho_{0} W^{2} \phi_{1} \phi_{2} d z, \\
v_{1} & =3 \int_{-h}^{0} \rho_{0} W^{2} \phi_{1 z}^{2} \phi_{2 z} d z, & v_{2} & =3 \int_{-h}^{0} \rho_{0} W^{2} \phi_{2 z}^{2} \phi_{1 z} d z, \\
I_{i} \gamma_{i} & =\tilde{f}^{2} \int_{-h}^{0} \rho_{0} \Phi_{i} \phi_{i z} d z, & \gamma_{i j} & =\tilde{f}^{2} \int_{-h}^{0} \rho_{0} \Phi_{i} \phi_{j z} d z .
\end{array}
$$

Here, $i, j=1,2$.

We scale the dependent and independent variables as

$$
A_{1}=\frac{u}{\mu_{1}}, \quad A_{2}=\frac{v}{\mu_{2}}, \quad s=\lambda_{1}^{1 / 2} X, \quad \tau=\lambda_{1}^{1 / 2} T,
$$


assuming that $\lambda_{2}>0, \lambda_{1} \neq 0, \mu_{1,2} \neq 0$ without the loss of generality. Then, Eqs. (42) and (43) take the form

$$
\begin{aligned}
& \left(u_{T}+u u_{X}+u_{X X X}+n(u v)_{X}+m v v_{X}+\alpha v_{X X X}\right)_{X}=\beta u+\gamma v, \\
& \left(v_{T}+v v_{X}+\delta v_{X X X}+\Delta v_{X}+p(u v)_{X}+q u u_{X}+\lambda u_{X X X}\right)_{X}=\mu v+v u,
\end{aligned}
$$

where

$$
\begin{aligned}
& n=\frac{v_{1}}{I_{1} \mu_{2}}, \quad m=\frac{\mu_{1} v_{2}}{I_{1} \mu_{2}^{2}}, \quad \alpha=\frac{\lambda_{12} \mu_{1}}{\lambda_{1} I_{1} \mu_{2}}, \quad \beta=\gamma_{1} \lambda_{1}, \quad \gamma=\frac{\gamma_{12} \mu_{1} \lambda_{1}}{I_{1} \mu_{2}} \\
& \delta=\frac{\lambda_{2}}{\lambda_{1}}, \quad p=\frac{\nu_{2}}{I_{2} \mu_{1}}, \quad q=\frac{\mu_{2} v_{1}}{I_{2} \mu_{1}^{2}}, \quad \lambda=\frac{\lambda_{21} \mu_{2}}{\lambda_{1} I_{2} \mu_{1}}, \quad \mu=\gamma_{2} \lambda_{1}, \quad v=\frac{\gamma_{21} \mu_{2} \lambda_{1}}{I_{2} \mu_{1}} .
\end{aligned}
$$

Here,

$$
\frac{q}{n}=\frac{p}{m}=\frac{\lambda}{\alpha}=\frac{\gamma_{12} v}{\gamma_{21} \gamma}=\frac{I_{1} \mu_{2}^{2}}{I_{2} \mu_{1}^{2}}, \quad \frac{\alpha \lambda}{\delta}=\frac{\lambda_{12}^{2}}{\lambda_{1} \lambda_{2} I_{1} I_{2}}<1 .
$$

Here, the scaled variables $u$ and $v$, and the coefficient $p$ should not be confused with the velocity components and the pressure. Note that with this scaling (48), the scaled variables $X, T$ have dimensions of $C^{-1 / 2}, C^{-3 / 2}$, respectively, where $C$ is a velocity scale, i.e., $\mathrm{m} \mathrm{s}^{-1}$. The dependent variables $u$ and $v$ have the dimension of $C$. The coefficients $n, m, \alpha, \delta, p, q, \lambda$ are dimensionless, while $\beta, \gamma, \mu, v$ have dimensions of $C^{2}$, and $\Delta$ has the dimension of $C$. The wavenumber $k$ and $c_{p}, c_{g}$ have the dimensions of $C^{1 / 2}$ and $C$, respectively. In the sequel, we omit writing these dimensions for the scaled variables but write the unscaled physical parameters in dimensional form.

\section{B. Three-layer flow with shear}

As an illustrative example with sufficient parameters to explore several cases of interest, we consider a three-layer background flow, $-h<z<0$, with interfaces at $z=-h_{2}-h_{1}, z=-h_{1}$, and $h=h_{1}+h_{2}+h_{3}$, shown in Figure 3. Here, $\rho_{0}$ and $u_{0}$ are piecewise-constant density and velocity fields, respectively, and they are represented using the Heaviside step-function as follows:

$$
\begin{aligned}
& \rho_{0}(z)=\rho_{3}+\left(\rho_{2}-\rho_{3}\right) H\left(z+h_{2}+h_{1}\right)+\left(\rho_{1}-\rho_{2}\right) H\left(z+h_{1}\right), \\
& u_{0}(z)=U_{3}+\left(U_{2}-U_{3}\right) H\left(z+h_{2}+h_{1}\right)+\left(U_{1}-U_{2}\right) H\left(z+h_{1}\right) .
\end{aligned}
$$

This three-layer flow model is not meant to be realistic in the strict sense but is used here as a guide for appropriate values of the parameters. The model is chosen to yield explicit formulae but can be regarded as a simplification of a background flow with smooth density and shear profiles across the interfaces. In the long wave limit we consider, we expect this piecewise model to yield coefficients close to those which would come from such a smooth model. It is also pertinent to note that this background flow is subject to Kelvin-Helmholtz instability, but these arise as short waves,

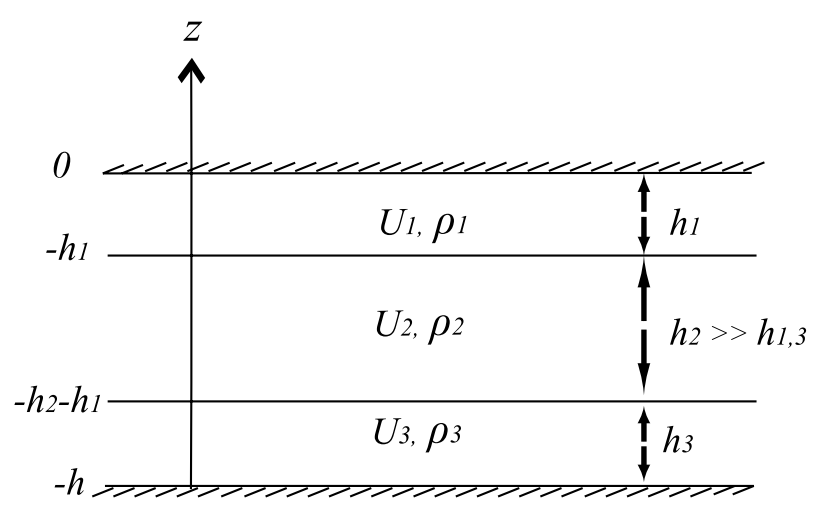

FIG. 3. A schematic representation of the three-layer model with shear flow. 
which may occur in reality, but are excluded in the long wave system we study here, due to the large separation of scales. With rigid boundaries at $z=-h, 0$, the modal functions are given by

$$
\begin{gathered}
\phi=A_{3} \frac{h+z}{h_{3}}, \quad-h<z<-h_{2}-h_{1}, \\
\phi=A_{1} \frac{h_{1}+h_{2}+z}{h_{2}}-A_{3} \frac{h_{1}+z}{h_{2}}, \quad-h_{2}-h_{1}<z<-h_{1}, \\
\phi=-A_{1} \frac{z}{h_{1}}, \quad-h_{1}<z<0 .
\end{gathered}
$$

The modal functions are normalized so that $\phi=A_{1,3}$ at $z=-h_{1},-h_{1}-h_{2}$. At each interface, there is the jump relation

$$
\left[\rho_{0}\left(c-u_{0}\right)^{2} \phi_{z}\right]=g\left[\rho_{0}\right] \phi
$$

This yields the system

$$
\begin{gathered}
\left\{\frac{\rho_{1}\left(c-U_{1}\right)^{2}}{h_{1}}+\frac{\rho_{2}\left(c-U_{2}\right)^{2}}{h_{2}}-g\left(\rho_{2}-\rho_{1}\right)\right\} A_{1}-\frac{\rho_{2}\left(c-U_{2}\right)^{2}}{h_{2}} A_{3}=0, \\
-\frac{\rho_{2}\left(c-U_{2}\right)^{2}}{h_{2}} A_{1}+\left\{\frac{\rho_{3}\left(c-U_{3}\right)^{2}}{h_{3}}+\frac{\rho_{2}\left(c-U_{2}\right)^{2}}{h_{2}}-g\left(\rho_{3}-\rho_{2}\right)\right\} A_{3}=0,
\end{gathered}
$$

which can be written as

$$
\begin{aligned}
& D_{1} A_{1}-E A_{3}=0, \quad-E A_{1}+D_{3} A_{3}=0, \\
& D_{1}=\frac{\rho_{1}\left(c-U_{1}\right)^{2}}{h_{1}}+\frac{\rho_{2}\left(c-U_{2}\right)^{2}}{h_{2}}-g\left(\rho_{2}-\rho_{1}\right), \\
& D_{3}=\frac{\rho_{3}\left(c-U_{3}\right)^{2}}{h_{3}}+\frac{\rho_{2}\left(c-U_{2}\right)^{2}}{h_{2}}-g\left(\rho_{3}-\rho_{2}\right), \\
& E=\frac{\rho_{2}\left(c-U_{2}\right)^{2}}{h_{2}} .
\end{aligned}
$$

Without the loss of generality, we put $U_{2}=0$ henceforth.

The dispersion relation, determining the speed $c$ is then given by

$$
D_{1} D_{3}=E^{2} \text {. }
$$

A resonance with two distinct modes requires that $D_{1}=D_{3}=E=0$ simultaneously. There are two cases, either $c=0$ or $h_{2} \gg h_{1,3}$. The first case contains implicit critical layers and hence is not considered here. The second case is

$$
h_{2} \gg h_{1,3}, \quad c=U_{1} \pm\left\{\frac{g h_{1}\left(\rho_{2}-\rho_{1}\right)}{\rho_{1}}\right\}^{1 / 2}=U_{3} \pm\left\{\frac{g h_{3}\left(\rho_{3}-\rho_{2}\right)}{\rho_{3}}\right\}^{1 / 2} .
$$

For given densities $\rho_{1,2,3}$ and layer depths $h_{1,3}$, these determine the allowed shear $U_{1}-U_{3}$. There are four cases; but in the sequel, we consider only the right-propagating waves, choosing $c>0$, which then impose a constraint on the allowed choices for $U_{1}-U_{3}$.

The modal functions and their derivatives are given by

$$
\begin{array}{ccc}
\phi_{1}=0, \quad \phi_{1 z}=0, & -h<z<-h_{2}-h_{1}, \\
\phi_{1}=\frac{h_{1}+h_{2}+z}{h_{2}}, \quad \phi_{1 z}=\frac{1}{h_{2}}, & -h_{2}-h_{1}<z<-h_{1}, \\
\phi_{1}=-\frac{z}{h_{1}}, \quad \phi_{1 z}=-\frac{1}{h_{1}}, & -h_{1}<z<0 ; \\
\phi_{2}=\frac{h+z}{h_{3}}, \quad \phi_{2 z}=\frac{1}{h_{3}}, & -h<z<-h_{2}-h_{1}, \\
\phi_{2}=-\frac{h_{1}+z}{h_{2}}, \quad \phi_{2 z}=-\frac{1}{h_{2}}, & -h_{2}-h_{1}<z<-h_{1},
\end{array}
$$




$$
\phi_{2}=0, \quad \phi_{2 z}=0, \quad-h_{1}<z<0 .
$$

Note that here the subscripts 1,2 on the modal functions should not be confused with the subscripts 1,2,3 for each layer. Now all coefficients in the coupled Ostrovsky equations can be calculated, taking into account that $h_{2} \gg h_{1,3}$, where appropriate

$$
\begin{aligned}
I_{1} \mu_{1} & =-\frac{3 \rho_{1}\left(c-U_{1}\right)^{2}}{h_{1}^{2}}, \quad I_{2} \mu_{2}=\frac{3 \rho_{3}\left(c-U_{3}\right)^{2}}{h_{3}^{2}}, \\
I_{1} \lambda_{1} & =I_{2} \lambda_{2}=\frac{c^{2} \rho_{2} h_{2}}{3}, \\
I_{1} & =\frac{2 \rho_{1}\left(c-U_{1}\right)}{h_{1}}, \quad I_{2}=\frac{2 \rho_{3}\left(c-U_{3}\right)}{h_{3}} \\
\lambda_{12} & =\lambda_{21}=\frac{c^{2} \rho_{2} h_{2}}{6}, \\
v_{1} & =v_{2}=0 .
\end{aligned}
$$

For the coefficients $\gamma_{1,2,12}$, we must evaluate $\Phi_{1,2}$

$$
\begin{aligned}
\Phi_{1,2} & =\phi_{1 z, 2 z}-\frac{\left(\rho_{0} u_{0}\right)_{z}}{\rho_{0} W} \phi_{1,2}, \\
I_{i} \gamma_{i} & =\tilde{f}^{2} \int_{-h}^{0} \rho_{0} \Phi_{i} \phi_{i z} d z, \\
\gamma_{i j} & =\tilde{f}^{2} \int_{-h}^{0} \rho_{0} \Phi_{i} \phi_{j z} d z .
\end{aligned}
$$

Here, $\rho_{0}, W=c-u_{0}$ are piecewise constant, so the second term in (75) behaves like a $\delta$-function. Specifically, write

$$
\Phi_{1,2}=\phi_{1 z, 2 z}+\left\{[\log |W|]_{z}-\frac{u_{0}}{W}\left[\log \rho_{0}\right]_{z}\right\} \phi_{1,2},
$$

where the last term can be ignored in the Boussinesq approximation, but is kept here, and we treat $\log |W|$ and $\log \rho_{0}$ as piecewise-constant functions. The derivatives of $[\cdots]$ are $\delta$-functions, leading to the product of a $\delta$-function with a discontinuous function in (76) and (77). In order to evaluate these expressions, we first note that $\phi_{1 z}, \phi_{2 z}$ are zero except in the upper and bottom layer, respectively, where they are constants, and also $\Phi_{1}=0$ in the bottom layer and $\Phi_{2}=0$ in the top layer. Hence,

$$
\begin{aligned}
& I_{1} \gamma_{1}=-\frac{\rho_{1} \tilde{f}^{2}}{h_{1}} \int_{-h}^{0} \Phi_{1} H\left(z+h_{1}\right) d z=\frac{\rho_{1} \tilde{f}^{2}}{h_{1}}\left\{1+\frac{1}{2} \log \left[\frac{\left|W_{2}\right|}{\left|W_{1}\right|}\right]-\frac{U_{1}}{2 W_{1}} \log \left[\frac{\rho_{2}}{\rho_{1}}\right]\right\}, \\
& I_{2} \gamma_{2}=\frac{\rho_{3} \tilde{f}^{2}}{h_{3}} \int_{-h}^{0} \Phi_{2} H\left(-z-h_{1}-h_{2}\right) d z=\frac{\rho_{3} \tilde{f}^{2}}{h_{3}}\left\{1+\frac{1}{2} \log \left[\frac{\left|W_{2}\right|}{\left|W_{3}\right|}\right]-\frac{U_{3}}{2 W_{3}} \log \left[\frac{\rho_{2}}{\rho_{3}}\right]\right\}, \\
& \gamma_{12}=\gamma_{21}=0 .
\end{aligned}
$$

Here, we have used the expression that when a $\delta$-function multiplies a discontinuous function $f(x)$,

$$
\int f(x) \delta(x) d x=\frac{1}{2}(f(0+)+f(0-)) .
$$

Next, we let $g_{1}=g\left(\rho_{2}-\rho_{1}\right) / \rho_{1}, g_{3}=g\left(\rho_{3}-\rho_{2}\right) / \rho_{3}$, and use the Boussinesq approximation that otherwise $\rho_{1} \approx \rho_{2} \approx \rho_{3}$. We then obtain that

$$
\begin{aligned}
& n=m=p=q=\gamma=v=0, \\
& \delta=\frac{h_{3}\left(c-U_{1}\right)}{h_{1}\left(c-U_{3}\right)}=-2 \alpha, \quad \lambda=-\frac{1}{2}, \\
& \beta=\frac{c^{2} h_{1} h_{2} \tilde{f}^{2}\left[1+\frac{1}{2} \log \left|c /\left(c-U_{1}\right)\right|\right]}{12\left(c-U_{1}\right)^{2}},
\end{aligned}
$$




$$
\begin{aligned}
\mu & =\frac{c^{2} h_{1} h_{2} \tilde{f}^{2}\left[1+\frac{1}{2} \log \left|c /\left(c-U_{3}\right)\right|\right]}{12\left(c-U_{1}\right)\left(c-U_{3}\right)} \\
\text { so that } \quad \mu & =\beta F, \quad F=\left\{\frac{c-U_{1}}{c-U_{3}}\right\} \frac{1+\frac{1}{2} \log \left|c /\left(c-U_{3}\right)\right|}{1+\frac{1}{2} \log \left|c /\left(c-U_{1}\right)\right|} .
\end{aligned}
$$

Then, there are four possibilities according to the value of $c$

$$
\begin{array}{ll}
\text { Case 1: } & c=U_{1}+\sqrt{g_{1} h_{1}}=U_{3}+\sqrt{g_{3} h_{3}}, \\
\text { Case 2: } & c=U_{1}-\sqrt{g_{1} h_{1}}=U_{3}-\sqrt{g_{3} h_{3}}, \\
\text { Case 3: } & c=U_{1}+\sqrt{g_{1} h_{1}}=U_{3}-\sqrt{g_{3} h_{3}}, \\
\text { Case 4: } & c=U_{1}-\sqrt{g_{1} h_{1}}=U_{3}+\sqrt{g_{3} h_{3}} .
\end{array}
$$

Bearing in mind that a piecewise-constant shear flow is a simplified model of a continuous shear flow, then in order to avoid an implicit critical layer, we choose $c>\max \left[U_{1}, 0, U_{3}\right]$, where we recall that we have set $U_{2}=0$. This condition then implies that only Case 1 is allowed.

In full detail, for Case 1,

$$
\begin{aligned}
& \delta=\sqrt{\frac{g_{1} h_{3}}{g_{3} h_{1}}}=-2 \alpha, \quad \lambda=-\frac{1}{2}, \\
& \beta=\frac{h_{2} \tilde{f}^{2}\left(\sqrt{g_{1} h_{1}}+U_{1}\right)^{2}\left[1+\frac{1}{2} \log \left|\left(\sqrt{g_{1} h_{1}}+U_{1}\right) / \sqrt{g_{1} h_{1}}\right|\right]}{12 g_{1}}, \\
& \mu=\beta F, \quad F=\sqrt{\frac{g_{1} h_{1}}{g_{3} h_{3}}} \frac{\left[1+\frac{1}{2} \log \left|\left(\sqrt{g_{3} h_{3}}+U_{3}\right) / \sqrt{g_{3} h_{3}}\right|\right]}{\left[1+\frac{1}{2} \log \left|\left(\sqrt{g_{1} h_{1}}+U_{1}\right) / \sqrt{g_{1} h_{1}}\right|\right]} .
\end{aligned}
$$

Note that $\beta>0$ unless $U_{1}$ is such that

$$
\left|1+\frac{U_{1}}{\sqrt{g_{1} h_{1}}}\right|<e^{-2}, \quad-1<\frac{U_{1}}{\sqrt{g_{1} h_{1}}}<e^{-2}-1=-0.865
$$

when $\beta<0$. Similarly, $\mu>0$ unless $U_{3}$ is such that

$$
\left|1+\frac{U_{3}}{\sqrt{g_{3} h_{3}}}\right|<e^{-2}, \quad-1<\frac{U_{3}}{\sqrt{g_{3} h_{3}}}<e^{-2}-1=-0.865
$$

when $\mu<0$. Here, we have used the condition for the exclusion of an implicit critical layer. Note also that $U_{1}, U_{3}$ are constrained by the resonance condition (86). Nevertheless, all four possibilities can be realised, that is, Case A: $\beta>0, \mu>0$, Case B: $\beta>0, \mu<0$, Case C: $\beta<0, \mu>0$, and Case $\mathrm{D}: \beta<0, \mu<0$.

Specifically, we choose $\tilde{f}=5 \times 10^{-3} \mathrm{~s}^{-1}$ and choose $g_{1,3}$ of the order $10^{-1} \leftrightarrow 10^{-3} \mathrm{~m} \mathrm{~s}^{-2}$. The upper layer and lower depths $h_{1,3}$ are chosen to be of order $50 \leftrightarrow 1000 \mathrm{~m}$. Next, we choose $U_{1}$ and use the resonance condition (86) to determine the value of $U_{3}$ since $U_{1}$ and $U_{3}$ are not independent. Finally, $h_{2}$ is a free parameter, so $\beta$ can be chosen arbitrarily, but then $\mu=\beta F$ is determined. Typically, we choose $\beta$ so that $h_{2} \gg h_{1,3}$ but of order $4 \leftrightarrow 6 \mathrm{~km}$. For instance, choose $U_{1}=1 \mathrm{~m} \mathrm{~s}^{-1}$, $h_{1}=50 \mathrm{~m}, g_{1}=0.1 \mathrm{~m} \mathrm{~s}^{-2}$, and then $\beta>0$; in this case, also $\mu>0$ when $\sqrt{g_{3} h_{3}}<23.97$ and $\mu<0$ when $\sqrt{g_{3} h_{3}}>23.97$, on using the resonance condition (86) to determine $U_{3}=3.236-\sqrt{g_{3} h_{3}}$. Alternatively, choose $U_{1}=-1.8 \mathrm{~m} \mathrm{~s}^{-1}, h_{1}=500 \mathrm{~m}, g_{1}=0.01 \mathrm{~m} \mathrm{~s}^{-2}$, and again $\beta>0$, but now $U_{3}=0.436-\sqrt{g_{3} h_{3}}$, so that $\mu<0$ when $\sqrt{g_{3} h_{3}}>3.22$, a more realistic value. Next, choose $U_{1}$ so that $-1<U_{1} / \sqrt{g_{1} h_{1}}<-0.865$, for instance $U_{1}=-1.8 \mathrm{~m} \mathrm{~s}^{-1}, h_{1}=800 \mathrm{~m}, g_{1}=0.005 \mathrm{~m} \mathrm{~s}^{-2}$, and then $\beta<0$; in this case, $U_{3}=0.2-\sqrt{g_{3} h_{3}}$ so that $\mu>0$ when $\sqrt{g_{3} h_{3}}<1.48$ and $\mu<0$ when $\sqrt{g_{3} h_{3}}>1.48$. Alternatively, we can choose $U_{1}=-1.4 \mathrm{~m} \mathrm{~s}^{-1}, h_{1}=1000 \mathrm{~m}, g_{1}=0.0025 \mathrm{~m} \mathrm{~s}^{-2}$, and then again $\beta<0$; but now $U_{3}=0.181-\sqrt{g_{3} h_{3}}$ so that $\mu>0$ when $\sqrt{g_{3} h_{3}}<1.34$ and $\mu<0$ when $\sqrt{g_{3} h_{3}}>1.34$. Although these velocities are quite large, note that they scale with $\sqrt{g_{1} h_{1}}$ and $\sqrt{g_{3} h_{3}}$ and would be somewhat smaller and more realistic if $g_{1}, g_{3}$ were reduced by a factor of $10^{-1}$ to $10^{-3}$. 
Finally in this section, we would like to point out that the type of the current model considered here can also lead to the anomalous version of the single Ostrovsky equation when $\lambda \gamma<0$. In particular, we show that the two-layer reduction of this three-layer model obtained by taking the $h_{2} \gg h_{1,3}$, that is, a single shallow layer with the density $\rho_{1}$ and current $U_{1}$ overlying a deep layer with the density $\rho_{2}$ and zero current can lead to this anomalous situation. Indeed, for this special case, the dispersion relation determining the speed $c$ is again given by (62), where we now let $h_{2} \gg h_{1,3}$, so that $E=0$, and then, for a single mode, either $D_{1}=0$ or $D_{3}=0$. Here, we choose $D_{1}=0$, and with now $D_{3} \neq 0$, it follows from (58) that $A_{3}=0, A_{1}$ is arbitrary and we set $A_{1}=1$. Then, the modal function $\phi$ obtained from (53) is given by

$$
\begin{gathered}
\phi=0, \quad \phi_{z}=0, \quad-h<z<-h_{2}-h_{1}, \\
\phi=\frac{h_{1}+h_{2}+z}{h_{2}}, \quad \phi_{z}=\frac{1}{h_{2}}, \quad-h_{2}-h_{1}<z<-h_{1}, \\
\phi=-\frac{z}{h_{1}}, \quad \phi_{z}=-\frac{1}{h_{1}}, \quad-h_{1}<z<0 .
\end{gathered}
$$

Without the loss of generality, we put $U_{2}=0$ henceforth. Then, using the limit $h_{2} \gg h_{1}$, the speed $c$ is given by

$$
c_{1,2}=U_{1} \pm\left(g^{\prime} h_{1}\right)^{1 / 2} \quad \text { where } \quad g^{\prime}=\frac{g\left(\rho_{2}-\rho_{1}\right)}{\rho_{1}} .
$$

Note that the third layer is not involved at all. Indeed, this analysis goes through in a similar manner when there is only one interface (the upper interface) and $h_{2}$ is finite, but we will not show the details here. To avoid an implicit critical layer, we must choose $c>\max \left[U_{1}, 0\right]$ or $c<\min \left[U_{1}, 0\right]$. The first case, denoted as the positive mode propagating to the right, holds provided that $U_{1}+\left(g^{\prime} h_{1}\right)^{1 / 2}>0$ and the latter, denoted as the negative mode propagating to the left, holds provided that $U_{1}-\left(g^{\prime} h_{1}\right)^{1 / 2}<0$.

Now all coefficients in the Ostrovsky equation (1) can be calculated, taking into account that $h_{2} \gg h_{1}$,

$$
\begin{aligned}
& I v=-\frac{3 \rho_{1} g^{\prime}}{h_{1}}, \\
& I \lambda=\frac{c^{2} \rho_{2} h_{2}}{3}, \\
& I \gamma=\frac{\rho_{1} \tilde{f}^{2}}{h_{1}}\left(1+\frac{1}{2} \log \frac{\left|W_{2}\right|}{\left|W_{1}\right|}-\frac{U_{1}}{2 W_{1}} \log \frac{\rho_{2}}{\rho_{1}}\right), \\
& I=\frac{2 \rho_{1} W_{1}}{h_{1}} .
\end{aligned}
$$

Note that $I>0$, so that $v<0, \lambda>0$, for the mode to the right and $I<0$, so that $v>0, \lambda<0$, for the mode to the left. As expected $v \lambda<0$ for both modes, which describe waves of depression. In the Boussinesq approximation when $\rho_{1} \approx \rho_{2}$, we obtain

$$
I \gamma=\frac{\rho_{1} \tilde{f}^{2}}{h_{1}}\left\{1+\frac{1}{2} \log \left[\frac{|c|}{\left|c-U_{1}\right|}\right]\right\} .
$$

Thus, $I \gamma>0$ unless $U_{1}$ is such that

$$
\begin{aligned}
\frac{|c|}{\sqrt{g h_{1}}}=\left|1 \pm \frac{U_{1}}{\sqrt{g h_{1}}}\right|<e^{-2}, \\
\text { that is, } \quad-1<\frac{U_{1}}{\sqrt{g_{1} h_{1}}}<e^{-2}-1=-0.865, \\
\text { or, } \quad 1>\frac{U_{1}}{\sqrt{g_{1} h_{1}}}>1-e^{-2}=0.865
\end{aligned}
$$




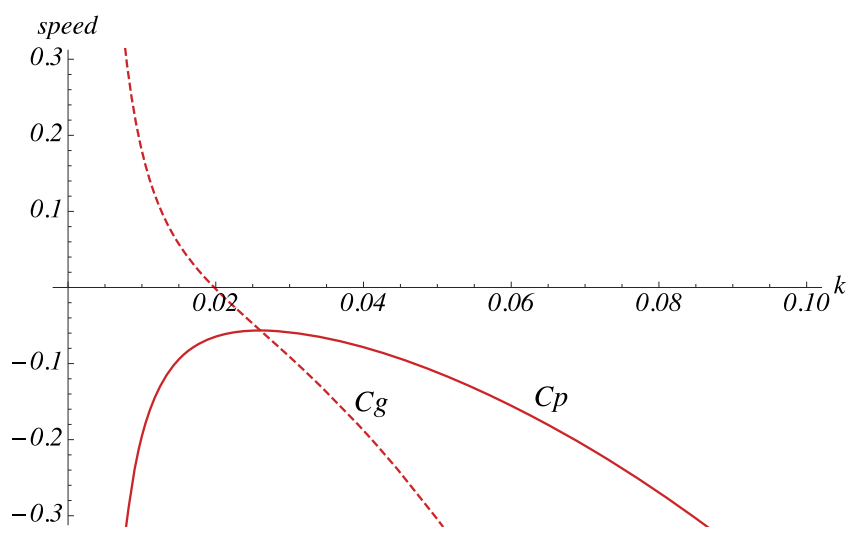

FIG. 4. Dispersion relation for the single Ostrovsky equation when $\lambda \gamma<0$.

for the mode to the right and left, respectively. Here, we have also used the condition for the exclusion of an implicit critical layer. Note that the two modes are essentially the same, so it is enough to consider the mode to the right. Then, unless (103) holds, $\lambda \gamma>0$ and we have the typical Ostrovsky equation with only unsteady wave packet solutions. But if instead (103) holds, then $\lambda \gamma<0$, and we have the anomalous Ostrovsky equation for which there is a steady envelope wave packet solution. Let us also note that in the case of a two-layer fluid with finite depths $h_{1}$ and $h_{2}$ as mentioned above, the condition (102) holds but $\mathrm{e}^{-2}$ is replaced with $\mathrm{e}^{-2 \kappa}$, where $\kappa=h /\left(h_{2}-h_{1}\right)$, yielding similar results.

A typical dispersion curve is shown in Figure 4, where $v=-4.7 \times 10^{-3}, \lambda=41.64$, and $\gamma=-1.9 \times 10^{-5}$, when setting $h_{1}=0.1 \mathrm{~km}, h_{2} \approx 3.0 \mathrm{~km}, U_{1}=-0.3 \mathrm{~m} \mathrm{~s}^{-1}, \rho_{1}=1 \mathrm{~kg} \mathrm{~m}^{-3}$, and $\rho_{2}=$ $1.0001 \mathrm{~kg} \mathrm{~m}^{-3}$. There exists a spectral gap for the phase speed, which has a maximum value $c=$ -0.057 at $k=0.026$. The group velocity is positive as $k \rightarrow 0$, but negative as $k \rightarrow \infty$, and at the point of maximum phase speed, the phase and group velocities are equal. Hence, a steady wave packet can exist.

A typical numerical result is shown in Figures 5 and 6 using a wave packet initial condition

$$
A(x, 0)=V_{0} A_{0} \operatorname{sech}\left(K_{0} X\right) \cos (k X),
$$

where $V_{0}=1, A_{0}=8, K_{0}=0.25 k$, and $k=0.026$. The solution is dominated by a steady wave packet, as expected, with the speed -0.069 , which is in good agreement with the theoretical value.

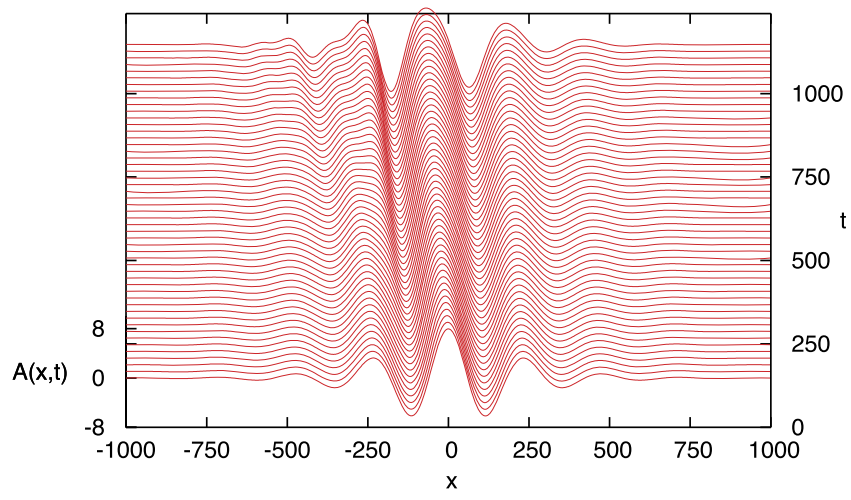

FIG. 5. Numerical simulation for the Ostrovsky equation (1) when $\lambda \gamma<0$ using the wave packet initial condition (105) with $k=0.026, A_{0}=8, K_{0}=0.25 k$, and $V_{0}=1$. 

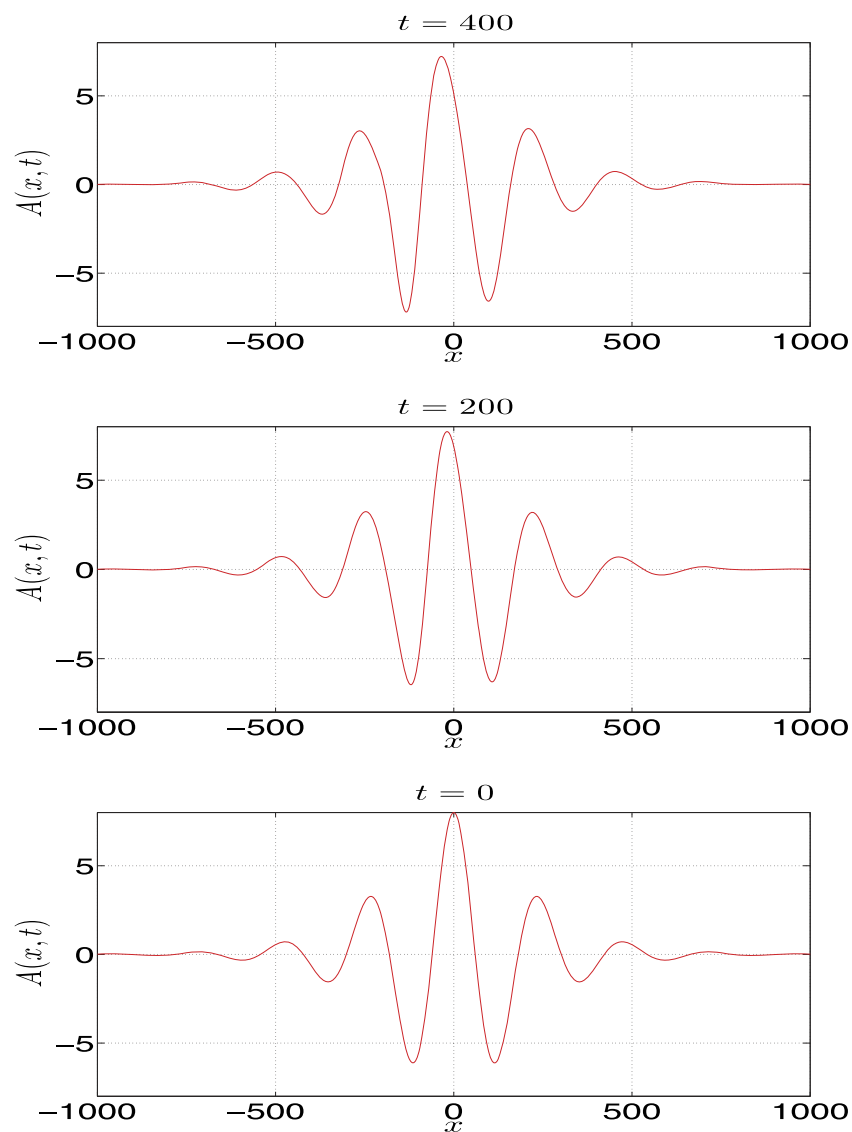

FIG. 6. Same as Figure 5 but a cross-section at $\tau=0,200,400$.

\section{LINEAR DISPERSION RELATION}

The structure of the linear dispersion relation determines the possible solution types. It is obtained by seeking solutions of the linearised equations in the form

$$
u=u_{0} e^{i k\left(X-c_{p} T\right)}+c . c ., \quad v=v_{0} e^{i k\left(X-c_{p} T\right)}+c . c .,
$$

where $k$ is the scaled wavenumber, $c_{p}(k)$ is the phase speed, and c.c. denotes the complex conjugate. This leads to

$$
\begin{aligned}
& \left(c_{p}-C_{1}(k)\right) u_{0}+\left(\alpha k^{2}-\frac{\gamma}{k^{2}}\right) v_{0}=0, \\
& \left(\lambda k^{2}-\frac{v}{k^{2}}\right) u_{0}+\left(c_{p}-C_{2}(k)\right) v_{0}=0,
\end{aligned}
$$

where $\quad C_{1}(k)=-k^{2}+\frac{\beta}{k^{2}}, \quad C_{2}(k)=\Delta-\delta k^{2}+\frac{\mu}{k^{2}}$.

The determinant of this $2 \times 2$ system yields the dispersion relation

$$
\left(c_{p}-C_{1}(k)\right)\left(c_{p}-C_{2}(k)\right)=D(k)=\left(\alpha k^{2}-\frac{\gamma}{k^{2}}\right)\left(\lambda k^{2}-\frac{v}{k^{2}}\right) .
$$

Solving this dispersion relation, we obtain the two branches of the dispersion relation

$$
c_{p}=c_{p 1, p 2}=\frac{C_{1}+C_{2}}{2} \pm \frac{1}{2}\left\{4 D+\left(C_{1}-C_{2}\right)^{2}\right\}^{1 / 2} .
$$

Here, $C_{1,2}(k)$ are the linear phase speeds of the uncoupled Ostrovsky equations, obtained formally by setting the coupling term $D(k)=0$. If $D(k)>0$ for all $k$, then both branches are real-valued for 
all wavenumbers $k$, and the linearised system is spectrally stable. Here, $\gamma=v=0$ and $\alpha \lambda>0$ so that $D(k)=\alpha \lambda k^{4}>0$ for all $k$.

Consider now Case 1 , where $c>0, I_{1}>0, I_{2}>0$, and so $\lambda_{1,2}>0$, so that $\delta>0$, and $0<\alpha \lambda$ $=\delta / 4$. Also, we recall that $\Delta<0$ without the loss of generality. The main effect of the background shear is that now $\beta \neq \mu$ and indeed each can be either positive or negative. Then, (111) takes the form

$$
c_{p}=c_{p 1, p 2}=\frac{\beta+\mu}{2 k^{2}}+\frac{\Delta}{2}-\frac{(1+\delta) k^{2}}{2} \pm \frac{1}{2} \sqrt{\left[\frac{\beta-\mu}{k^{2}}-\Delta-(1-\delta) k^{2}\right]^{2}+4 \alpha \lambda k^{4}} .
$$

The group velocities are given by $c_{g}=d\left(k c_{p}\right) / d k$

$$
\begin{aligned}
c_{g}=c_{g 1, g 2}= & -\frac{\beta+\mu}{2 k^{2}}+\frac{\Delta}{2}-\frac{3(1+\delta) k^{2}}{2} \\
& \pm \frac{\left(\Delta+(1-\delta) k^{2}-\frac{\beta-\mu}{k^{2}}\right)\left(\frac{3}{2}(1-\delta) k^{2}+\frac{1}{2}\left(\Delta+\frac{\beta-\mu}{k^{2}}\right)\right)+6 \alpha \lambda k^{4}}{\sqrt{\left(\Delta+(1-\delta) k^{2}-\frac{\beta-\mu}{k^{2}}\right)^{2}+4 \alpha \lambda k^{4}}} .
\end{aligned}
$$

Next, it is useful to examine the limits $k \rightarrow 0, \infty$. Thus,

$$
\begin{array}{cc}
c_{p 1, p 2} \rightarrow \frac{F_{1,2}}{k^{2}}, 2 F_{1,2}=\beta+\mu \pm|\beta-\mu| & \text { as } \quad k \rightarrow 0, \\
c_{p 1, p 2} \rightarrow E_{1,2} k^{2}, 2 E_{1,2}=-(1+\delta) \pm\left\{(1-\delta)^{2}+4 \alpha \lambda\right\}^{1 / 2} & \text { as } \quad k \rightarrow \infty . \\
c_{g 1, g 2} & \rightarrow-\frac{F_{1,2}}{k^{2}} \quad \text { as } \quad k \rightarrow 0, \\
c_{g 1, g 2} & \rightarrow 3 E_{1,2} k^{2} \quad \text { as } \quad k \rightarrow \infty .
\end{array}
$$

Note that since $0<\alpha \lambda<\delta, E_{2}<E_{1}<0$. One can see that there are four possibilities of qualitatively different behaviours of the dispersion relation, depending on the signs of the coefficients $\beta$ and $\mu$, as Case A: $\beta>0, \mu>0$, Case B: $\beta>0, \mu<0$, Case C: $\beta<0, \mu>0$, and Case D: $\beta<0, \mu<0$.

Case A: $\beta>0, \mu>0$. Then, $F_{1}=\max [\beta, \mu]>F_{2}=\min [\beta, \mu]>0$. There is no spectral gap in either mode, and this case is similar to the situation without any background shear, discussed in our previous paper. ${ }^{19}$ But there is now a significant difference since here $\beta \neq \mu$ due to the effect of the background shear flow. A typical dispersion curve is shown in Figure 7, where $\beta=1, \mu=0.604, \Delta=-0.5, \delta=1.414, \alpha=-0.707$, and $\lambda=-0.5$ when setting $h_{1}=50 \mathrm{~m}, h_{2}$ $\approx 3.9 \mathrm{~km}, h_{3}=100 \mathrm{~m}, g_{1}=g_{3}=0.1 \mathrm{~m} \mathrm{~s}^{-2}, U_{1}=1 \mathrm{~m} \mathrm{~s}^{-1}, U_{3}=0.074 \mathrm{~m} \mathrm{~s}^{-1}, \rho_{1}=0.99 \rho_{2}$, and $\rho_{3}$ $=1.01 \rho_{2}$. Here, and in the subsequent plots of dispersion curves, the letters $A, B, \ldots$ indicate the turning points and possible resonant points, identified for comparison with our numerical results. For both modes, the group velocities are negative for all $k$ and each has a single turning point

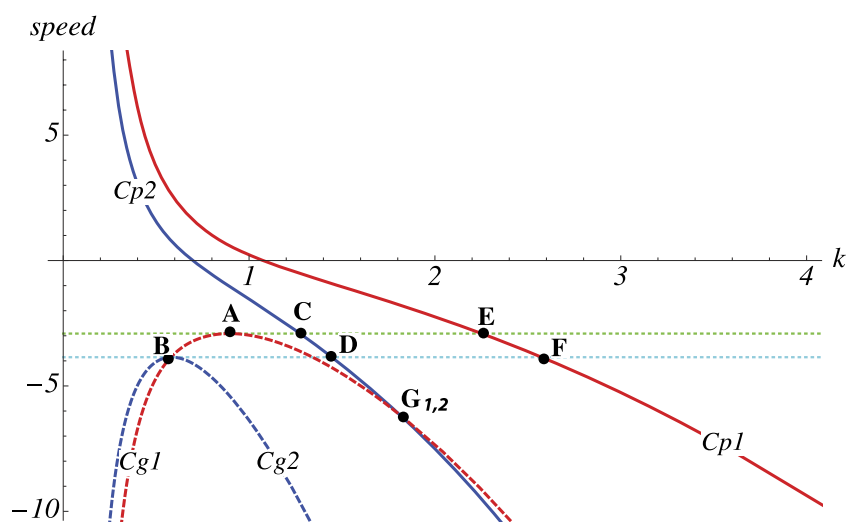

FIG. 7. Typical dispersion curve for Case A with $\delta=1.414, \alpha=-0.707, \lambda=-0.5, \Delta=-0.5, \beta=1$, and $\mu=0.604$. 


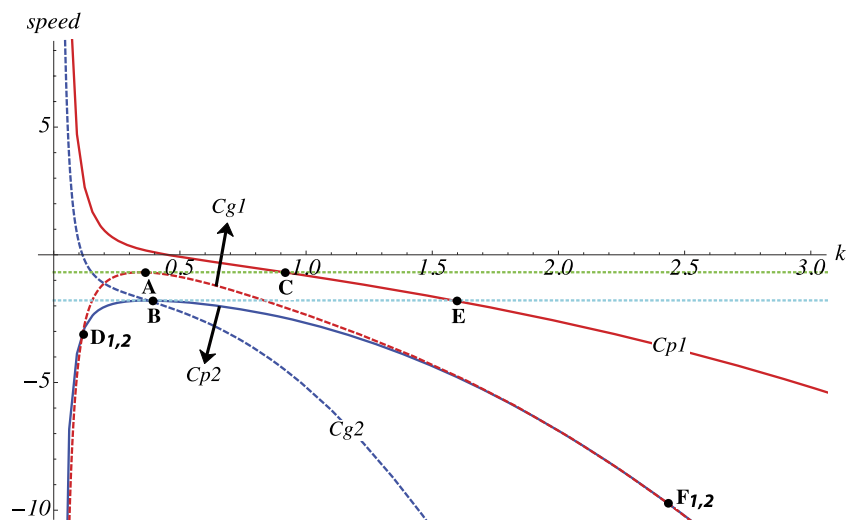

FIG. 8. Typical dispersion curve for Case B with $\delta=1, \alpha=-0.5, \lambda=-0.5, \Delta=-1.5, \beta=0.04$, and $\mu=-0.02$.

at $k=k_{m 1, m 2}$, respectively. In general, it is possible that there are $0,2,4, \ldots$ turning points for $c_{p}$ where $d c_{p} / d k=0$ and $c_{p}=c_{g}$. Each such turning point can generate a generalised envelope solitary wave, see Grimshaw and Iooss ${ }^{22}$ for instance. Further, it is also possible that there are $1,3,5, \ldots$ turning points for $c_{g}$ where $d c_{g} / d k=0$ and each such turning point is expected to generate an unsteady wave packet analogous to those found by Grimshaw and Helfrich ${ }^{11}$ for the single Ostrovsky equation. Figure 2 shows the simplest case when there are 0,1 turning points, respectively. But since there are four independent parameters $\beta, \mu, \Delta, \alpha \lambda$ (note that $\delta=-2 \alpha, \lambda=-0.5$, see (90)) in the expressions (112) and (113) for $c_{p}, c_{g}$, respectively, we cannot rule out the possibility that other "non-typical" cases may occur. Even though the expressions (112) and (113) are explicit, a full exploration of the 4-dimensional parameter space is beyond our present scope. Nevertheless, an asymptotic expansion in the parameter $\alpha \lambda \ll 1$ described below confirms that only the typical case arises in this asymptotic regime.

Case B: $\beta>0, \mu<0$. Then, $F_{1}=\beta>0, F_{2}=\mu<0$. A typical dispersion curve is shown in Figure 8 , where $\beta=0.04, \mu=-0.02, \Delta=-1.5, \delta=1, \alpha=-0.5$, and $\lambda=-0.5$ when setting $h_{1}=500 \mathrm{~m}$, $h_{2} \approx 5.5 \mathrm{~km}, h_{3}=1000 \mathrm{~m}, g_{1}=0.01 \mathrm{~m} \mathrm{~s}^{-2}, g_{3}=0.02 \mathrm{~m} \mathrm{~s}^{-2}, U_{1}=-1.8 \mathrm{~m} \mathrm{~s}^{-1}, U_{3}=-4.036 \mathrm{~m} \mathrm{~s}^{-1}$, $\rho_{1}=0.999 \rho_{2}$, and $\rho_{3}=1.002 \rho_{2}$. There is no spectral gap in mode 1 , and the group velocity is negative for all $k$ with a turning point at $k=k_{m 1}$. But mode 2 has a spectral gap, as the phase speed has a maximum value, $c_{s 2}$ at $k=k_{s 2}$. For this mode, the group velocity is positive as $k \rightarrow 0$ and negative as $k \rightarrow \infty$. At the value $c_{p 2}=c_{s 2}$, the phase and group velocities are equal, and then this mode 2 can support a steady wave packet. However, this wave packet lies in the spectrum of mode 1 and hence may decay by radiation into mode 1 ; strictly, it is a generalised solitary wave. Here, in general, it is possible that there are $0,2,4, \ldots$ turning points for $c_{p}$ for mode 1 and $1,3,5, \ldots$ for mode 2 . Further, it is also possible here that there are $1,3,5, \ldots$ turning points for $c_{g}$ in mode 1 and $0,2,4, \ldots$ for mode 2 . However, the asymptotic expansion in the parameter $\alpha \lambda \ll 1$ described below confirms that only the typical case $0,1,1,0$ of turning points arises in this asymptotic regime.

Case C: $\beta<0, \mu>0$. Then, $F_{1}=\mu>0, F_{2}=\beta<0$. A typical dispersion curve set is shown in Figure 9 , where $\beta=-0.01, \mu=0.002, \Delta=-0.1, \delta=1.414, \alpha=-0.707$, and $\lambda=-0.5$ when setting $h_{1}=h_{3}=800 \mathrm{~m}, h_{2} \approx 4.0 \mathrm{~km}, g_{1}=0.005 \mathrm{~m} \mathrm{~s}^{-2}, g_{3}=0.0025 \mathrm{~ms}^{-2}, U_{1}=-1.8 \mathrm{~m} \mathrm{~s}^{-1}, U_{3}=-1.214$ $\mathrm{m} \mathrm{s}^{-1}, \rho_{1}=0.9995 \rho_{2}$, and $\rho_{3}=1.00025 \rho_{2}$. At first glance, this is overall similar to Case B because there is no spectral gap in mode 1 , and the group velocity is negative for all $k$; but now the group velocity $c_{g 1}$ has three turning points, a global maximum at $A$, a local minimum at $K$, and a local maximum at $B$. This is not the simplest case, where we would expect only one turning point, but we display it here as potentially there could be energy focusing associated with each of these turning points and the consequent emergence of three unsteady nonlinear wave packets. As in Case B, mode 2 has a spectral gap as the phase speed has a maximum at $C$; the group velocity is positive as $k \rightarrow 0$ and negative as $k \rightarrow \infty$. At this point, the phase and group velocities are equal, and so then this mode 2 can support a steady wave packet. However, this wave packet lies in the spectrum of mode 1 and hence may decay by radiation into mode 1 . 


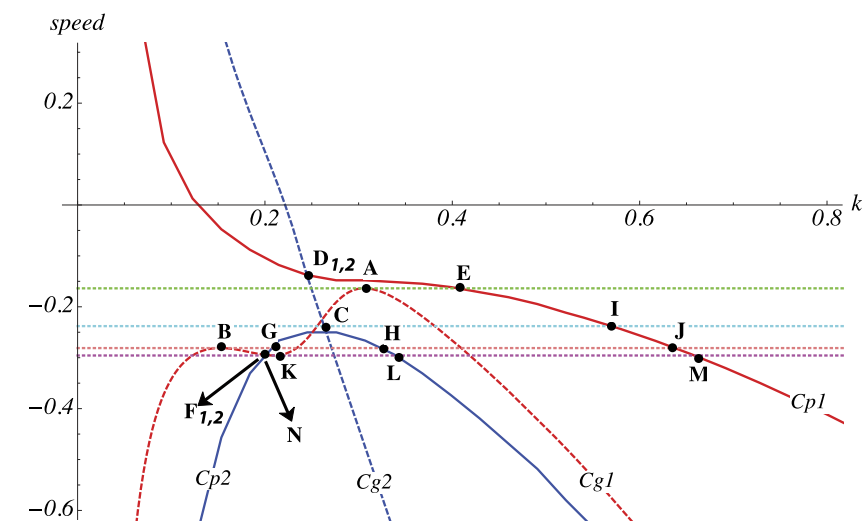

FIG. 9. Typical dispersion curve for Case $\mathrm{C}$ with $\delta=1.414, \alpha=-0.707, \lambda=-0.5, \Delta=-0.1, \beta=-0.01$, and $\mu=0.002$.

Case D: $\beta<0, \mu<0$. Then $F_{2}=\min [\beta, \mu]<F_{1}=\max [\beta, \mu]<0$. A typical dispersion curve for this case is shown in Figure 10, where $\beta=-0.01, \mu=-0.02, \Delta=-0.5, \delta=0.707, \alpha=-0.354$, and $\lambda=-0.5$ when setting $h_{1}=h_{3}=1000 \mathrm{~m}, h_{2} \approx 4.4 \mathrm{~km}, g_{1}=0.0025 \mathrm{~m} \mathrm{~s}^{-2}, g_{3}=0.005 \mathrm{~m} \mathrm{~s}^{-2}$, $U_{1}=-1.4 \mathrm{~m} \mathrm{~s}^{-1}, U_{3}=-2.055 \mathrm{~m} \mathrm{~s}^{-1}, \rho_{1}=0.9998 \rho_{2}$, and $\rho_{3}=1.0005 \rho_{2}$. Now both modes have phase speeds with maxima $c_{s 1}, c_{s 2}$ at $k=k_{s 1}, k_{s 2}$, denoted by the points $A, B$, respectively. For both modes, the group velocity is positive as $k \rightarrow 0$, but negative as $k \rightarrow \infty$, and at the point of maximum phase speed, the phase and group velocities for each mode are equal. Hence, a steady wave packet can exist for each mode but will be radiating for mode 2 .

As indicated above, we use an asymptotic expansion with $\epsilon=\alpha \lambda \ll 1$ to find all turning points explicitly. From (110), since here $\gamma=v=0$,

$$
\left(c_{p}-C_{1}(k)\right)\left(c_{p}-C_{2}(k)\right)=\epsilon k^{4}, \quad C_{1}(k)=-k^{2}+\frac{\beta}{k^{2}}, \quad C_{2}(k)=\Delta-\delta k^{2}+\frac{\mu}{k^{2}},
$$

where $\epsilon=\alpha \lambda \ll 1$. Note that the effective expansion parameter is $\epsilon k^{4}$ and so this can only be valid when $k$ is also sufficiently small, say $k<1$. Expanding in powers of $\epsilon$ then yields

$$
\begin{aligned}
& c_{p a}=C_{1}+\frac{\epsilon k^{4}}{C_{1}-C_{2}}-\frac{\epsilon^{2} k^{8}}{\left(C_{1}-C_{2}\right)^{3}}+\cdots, \\
& c_{p b}=C_{2}-\frac{\epsilon k^{4}}{C_{1}-C_{2}}+\frac{\epsilon^{2} k^{8}}{\left(C_{1}-C_{2}\right)^{3}}+\cdots .
\end{aligned}
$$

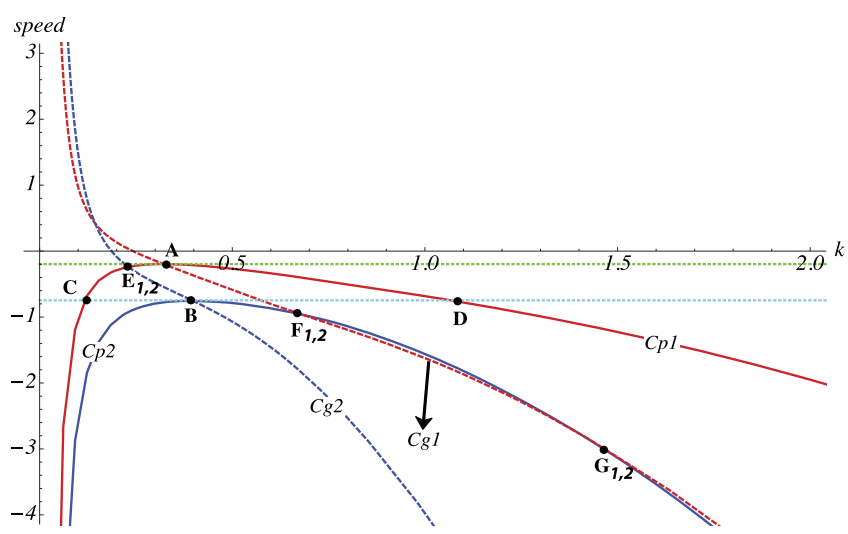

FIG. 10. Typical dispersion curve for Case D with $\delta=0.707, \alpha=-0.354, \lambda=-0.5, \Delta=-0.5, \beta=-0.01$, and $\mu=-0.02$. 
The derivatives are given by

$$
\begin{aligned}
& c_{p a k}=-2 k-\frac{2 \beta}{k^{3}}+\epsilon\left\{\frac{4 k^{3}}{C_{1}-C_{2}}-\frac{k^{4}\left(C_{1 k}-C_{2 k}\right)}{\left(C_{1}-C_{2}\right)^{2}}\right\}+\cdots, \\
& c_{p b k}=-2 \delta k-\frac{2 \mu}{k^{3}}-\epsilon\left\{\frac{4 k^{3}}{C_{1}-C_{2}}-\frac{k^{4}\left(C_{1 k}-C_{2 k}\right)}{\left(C_{1}-C_{2}\right)^{2}}\right\}+\cdots .
\end{aligned}
$$

The corresponding group velocities are found from $c_{g}=c_{p}+k c_{p k}$

$$
\begin{aligned}
& c_{g a}=-3 k^{2}-\frac{\beta}{k^{2}}+\epsilon\left\{\frac{5 k^{4}}{C_{1}-C_{2}}-\frac{k^{5}\left(C_{1 k}-C_{2 k}\right)}{\left(C_{1}-C_{2}\right)^{2}}\right\}+\cdots, \\
& c_{g b}=\Delta-3 \delta k^{2}-\frac{\mu}{k^{2}}-\epsilon\left\{\frac{5 k^{4}}{C_{1}-C_{2}}-\frac{k^{5}\left(C_{1 k}-C_{2 k}\right)}{\left(C_{1}-C_{2}\right)^{2}}\right\}+\cdots, \\
& c_{g a k}=-6 k+\frac{2 \beta}{k^{3}}+\epsilon\left\{\frac{20 k^{3}}{C_{1}-C_{2}}-\frac{10 k^{4}\left(C_{1 k}-C_{2 k}\right)}{\left(C_{1}-C_{2}\right)^{2}}-k^{5}\left\{\frac{\left(C_{1 k}-C_{2 k}\right)}{\left(C_{1}-C_{2}\right)^{2}}\right\}_{k}\right\}+\cdots, \\
& c_{g b k}=-6 \delta k+\frac{2 \mu}{k^{3}}-\epsilon\left\{\frac{20 k^{3}}{C_{1}-C_{2}}-\frac{10 k^{4}\left(C_{1 k}-C_{2 k}\right)}{\left(C_{1}-C_{2}\right)^{2}}-k^{5}\left\{\frac{\left(C_{1 k}-C_{2 k}\right)}{\left(C_{1}-C_{2}\right)^{2}}\right\}_{k}\right\}+\cdots .
\end{aligned}
$$

The turning points for $c_{p}$ can now be found by equating (120) and (121) to zero and those for $c_{g}$ found by equating (124) and (125) to zero. Consistently with this asymptotic expansion, the solutions for $k$ are sought in the form $k=k_{0}+\epsilon k_{1}+\epsilon^{2} k_{2}+\cdots$ by collecting the $O(1)$ and $O(\epsilon)$ terms. Then, we obtain the following formal asymptotic solutions:

$$
\begin{aligned}
c_{p a k}=0: \quad k & =k_{0}+\epsilon \frac{k_{0}^{9}\left(k_{0}^{4}(-1+\delta)-2 k_{0}^{2} \Delta+3(\beta-\mu)\right)}{\left(k_{0}^{4}-3 \beta\right)\left(\beta+k_{0}^{4}(-1+\delta)-k_{0}^{2} \Delta-\mu\right)^{2}}+\cdots \quad, k_{0}=\sqrt[4]{-\beta} ; \\
c_{p b k}=0: \quad k & =k_{0}-\epsilon \frac{k_{0}^{9}\left(k_{0}^{4}(-1+\delta)-2 k_{0}^{2} \Delta+3(\beta-\mu)\right)}{\left(k_{0}^{4} \delta-3 \mu\right)\left(\beta+k_{0}^{4}(-1+\delta)-k_{0}^{2} \Delta-\mu\right)^{2}}+\cdots \quad, k_{0}=\sqrt[4]{\frac{-\mu}{\delta}} ; \\
c_{g a k}=0: \quad k & =k_{0}+\epsilon k_{0}^{9}\left\{\frac{3 k_{0}^{8}(-1+\delta)^{2}-9 k_{0}^{6}(-1+\delta) \Delta+21(\beta-\mu)^{2}+27 k_{0}^{2} \Delta(-\beta+\mu)}{3\left(k_{0}^{4}+\beta\right)\left(\beta+k_{0}^{4}(-1+\delta)-k_{0}^{2} \Delta-\mu\right)^{3}}\right. \\
& \left.+\frac{2 k_{0}^{4}\left(4 \beta(-1+\delta)+5 \Delta^{2}+4 \mu(1-\delta)\right.}{3\left(k_{0}^{4}+\beta\right)\left(\beta+k_{0}^{4}(-1+\delta)-k_{0}^{2} \Delta-\mu\right)^{3}}\right\}+\cdots \quad, k_{0}=\sqrt[4]{\frac{\beta}{3}} ; \\
c_{g b k}=0: \quad k & =k_{0}-\epsilon k_{0}^{9}\left\{\frac{3 k_{0}^{8}(-1+\delta)^{2}-9 k_{0}^{6}(-1+\delta) \Delta+21(\beta-\mu)^{2}+27 k_{0}^{2} \Delta(-\beta+\mu)}{3\left(k_{0}^{4} \delta+\mu\right)\left(\beta+k_{0}^{4}(-1+\delta)-k_{0}^{2} \Delta-\mu\right)^{3}}\right. \\
& \left.-\frac{2 k_{0}^{4}\left(4 \beta(-1+\delta)+5 \Delta^{2}+4 \mu(1-\delta)\right.}{3\left(k_{0}^{4} \delta+\mu\right)\left(\beta+k_{0}^{4}(-1+\delta)-k_{0}^{2} \Delta-\mu\right)^{3}}\right\}+\cdots \quad, k_{0}=\sqrt[4]{\frac{\mu}{3 \delta}} .
\end{aligned}
$$

The outcomes for each case are described below.

Case A: $\beta>0, \mu>0$. Here, we put $a=1, b=2$ and find that both $c_{p 1 k}<0$ and $c_{p 2 k}<0$. Thus, there are no turning points for $c_{p 1}$ and $c_{p 2}$ in this approximation. However, $c_{g 1 k}=0$ yields just one turning point $k=k_{0}+\epsilon k_{1} \approx 0.868$ for the parameter values of Figure 7, compared to the exact value 0.895 . Also, $c_{g 2 k}=0$ yields just one turning point $k=k_{0}+\epsilon k_{1} \approx 0.574$, compared to the exact value 0.584 .

Case B: $\beta>0, \mu<0$. Here, we again put $a=1, b=2$ and find that $c_{p 1 k}<0$ and so there is no turning point for $c_{p 1}$. However, there is a single turning point for $c_{p 2}$, given by $c_{p 2 k}=0$, $k=k_{0}+\epsilon k_{1} \approx 0.372$, for the parameter values of Figure 8 compared to the exact value 0.372 . Next, there is a single turning point for $c_{g 1}$ when $c_{g 1 k}=0$ gives $k=k_{0}+\epsilon k_{1} \approx 0.345$, compared to the exact value 0.345 . Since $c_{g 2 k}<0$, there are no turning points for $c_{g 2}$. 
Case C: $\beta<0, \mu>0$. Here we put $a=2, b=1$ and find that $c_{p 1 k}<0$ and so there is no turning point for $c_{p 1}$. However, there is a single turning point for $c_{p 2}$, given by $c_{p 2 k}=0, k=k_{0}+\epsilon k_{1} \approx-0.408$ for the parameter values of Figure 9, compared to the exact value of 0.259. However, we note here that $k_{0}=0.316$ and the correction term $\left|\epsilon k_{1}\right|$ is much too large, indicating that the asymptotic expansion is not at all useful in this case. Next, there is a single turning point for $c_{g 1}$ and $c_{g 1 k}=0$ gives $k=k_{0}+\epsilon k_{1} \approx 0.151$, compared to the exact value of 0.152 , that is, point $B$ in Figure 9. However, we note here there also exists a minimum point $K$ in Figure 9 at $k=0.209$ and a maximum point $A$ at $k=0.306$ which are not found by this asymptotic analysis. Since $c_{g 2 k}<0$, there are no stationary points in $c_{g 2}$.

Case D: $\beta<0, \mu<0$. Here, we put $a=1, b=2$. There are turning points for both $c_{p 1}, c_{p 2}$ and $c_{p 1 k}$ $=0, c_{p 2 k}=0$ yield $k=k_{0}+\epsilon k_{1} \approx 0.322,0.392$, respectively, for the parameter values of Figure 10, compared to the exact values of $0.322,0.395$. Here, both $c_{g 1 k}<0$ and $c_{g 2 k}<0$, and hence there are no turning points in both $c_{g 1}$ and $c_{g 2}$.

\section{NUMERICAL SIMULATIONS}

In this section, we present some results from numerical simulations of the scaled equations (49) and (50), using the pseudo-spectral method described in Alias et al. ${ }^{19}$ for the four different cases, corresponding to the parameters of the linear dispersion curves described in Sec. III. We note again that in these equations, $X, T$ are scaled variables, see (48), and have dimensions of $C^{-1 / 2}, C^{-3 / 2}$, respectively, where $C$ is the velocity scale. The dependent variables $u$ and $v$ have the dimension of $C$. The coefficients $n, m, \alpha, \delta, p, q, \lambda$ are dimensionless, while $\beta, \gamma, \mu, v$ have dimensions of $C^{2}$, and $\Delta$ has the dimension of $C$. For all cases considered here, we have

$$
n=m=p=q=\gamma=v=0 .
$$

For the initial conditions, we use either an approximation to a solitary wave solution of the corresponding coupled $\mathrm{KdV}$ system, which is mainly suitable for Case A, or an approximation to a nonlinear wave packet, which is more suitable for Cases B, C, and D. The former initial condition is described by Alias et al., ${ }^{19}$ is denoted as "weak coupling KdV solitary waves," and given by

$$
\begin{array}{ll}
u=a \operatorname{sech}^{2}\left(\gamma_{1} X\right), & \frac{a}{3}=4(1+\alpha) \gamma_{1}^{2}, \\
v=b \operatorname{sech}^{2}\left(\gamma_{2} X\right), & \frac{b}{3}=4(\delta+\lambda) \gamma_{2}^{2} .
\end{array}
$$

This was mostly implemented with the constraint that $\gamma_{1}=\gamma_{2}$. Note that here the nonlinear terms $\left(u^{2} / 2\right)_{X X},\left(v^{2} / 2\right)_{X X}$ have maximum absolute values of $2 a^{2} \gamma_{1}^{2}=a^{3} / 6(1+\alpha)$ and $2 b^{2} \gamma_{2}^{2}=b^{3} / 6(\delta$ $+\lambda)$, respectively.

The nonlinear wave packet initial condition is based on either a maximum point in the group velocity curve where $\partial c_{g} / \partial k=0$ and $k=k_{m}$, or a maximum point in the phase velocity curve where $c_{p}=c_{g}$ and $k=k_{s}$. The former corresponds to the unsteady nonlinear wave packet travelling at a speed close to the maximum group velocity, and is relevant for both modes in Case A, but only for mode 1 in Cases B and C. The latter corresponds to a steady wave packet and is relevant for mode 2 in Cases B and C, and both modes in Case D.

To obtain a suitable wave packet initial condition, the procedure is to choose $k$, either $k_{m}$ or $k_{s}$, and then find the ratio $r=u_{0} / v_{0}$ from (107) or (108) in the form $u_{0}=U_{0} a_{0}, v_{0}=V_{0} a_{0}$ where $a_{0}$ is an arbitrary function of $X$, but $U_{0}, V_{0}$ are known functions of $k$. Based on the expected outcome that the nonlinear wave packet will be governed by an evolution equation such as the nonlinear Schrödinger equation, we choose $a_{0}(X)=A_{0} \operatorname{sech}\left(K_{0} X\right)$. Note that the underlying theory suggests that the shape should be sech and that $K_{0}$ depends on the amplitude $A_{0}$ (e.g., Grimshaw and Helfrich ${ }^{11}$ ). Here, instead we choose a value of $K_{0}<<k$. Then, the wave packet initial condition is

$$
u(X, 0)=r V_{0} A_{0} \operatorname{sech}\left(K_{0} X\right) \cos (k X), \quad v(X, 0)=V_{0} A_{0} \operatorname{sech}\left(K_{0} X\right) \cos (k X),
$$

where $r=U_{0} / V_{0}$ is a known function of $k$, and we can choose $V_{0}$ arbitrarily, say $V_{0}=1$.

Our main aim is to understand and interpret the observed dynamical behaviour by relating it to the main features of the relevant dispersion curves, comparing especially the theoretically predicted 

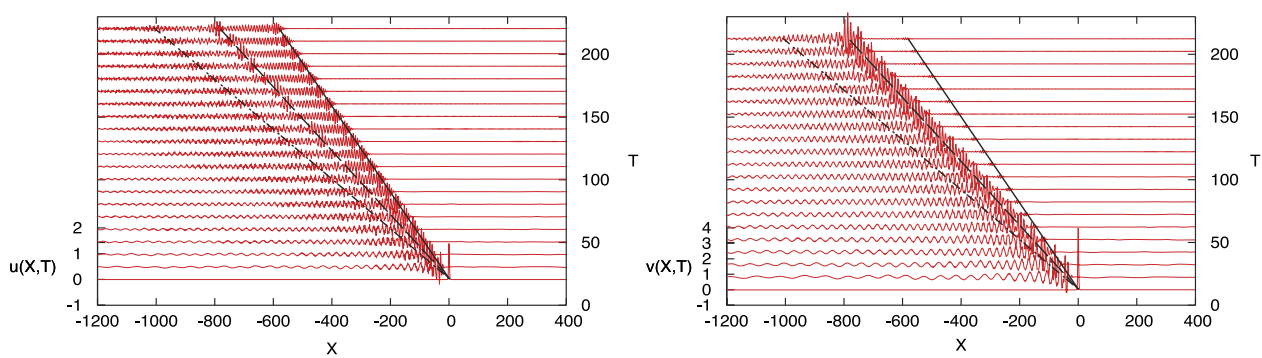

FIG. 11. Numerical simulations for Case A using a KdV initial condition of weak coupling with $a=1.4$ and $b=4.38$ in (126). The solid, dashed, and dashed-dotted lines in both plots refer to the points $A, B$, and $G_{1}$ in Figure 7 .

group speeds and $r=u_{0} / v_{0}$ amplitude ratios with those found in the numerical simulations. For the latter, we adopt the following methodology; the speed is measured at the maximum of the dominant wave packet, and the numerical ratio is measured as $R=\max |u| / \max |v|$ in the interval between the two nearest peaks, containing the maximum value of the dominant wave packet. Note that $R$ is necessarily positive, unlike $r$, since phase determination numerically is quite difficult. In some cases, wave packets generated in the numerical simulations are either contaminated by radiation or show signs of more than one carrier wavelength. In these cases, the ratio is not so instructive, and instead we choose the relevant points on the dispersion curves primarily by the speed of the wave packet, ruling out some points if the corresponding wavelength is too long or too short.

\section{A. Numerical results}

Case A: A typical numerical result is shown in Figures 11 and 12 using the KdV solitary wave initial condition (126). The generation of two wave packets can be seen in the $u$-component, but one of them is too small to be seen in the $v$-component. The comparison of the numerical modal ratio, $R$ determined as described above, shows very good agreement with the theoretical prediction from the dispersion relation, see Table I. The theoretical modal ratio is $r=3.692$ for mode 1 and $r=-0.132$ for mode 2, while the speeds are $c_{g 1}=-2.912, c_{g 2}=-3.854$ and $k_{m 1}=0.895, k_{m 2}=0.584$. The ratios of the numerically found wave packets obtained from the vertical dashed lines $A$ and $B$ in Figure 12 are given, respectively, by $R=3.433$ for mode 1 and $R=0.176$ for mode 2 , which are in agreement with the theoretical predictions, and the numerically found speeds $-2.960,-3.933$ are also in good agreement. However, we see that there is also some significant radiation to the left of these wave packets, and in particular some focusing possibly associated with the point $G_{1}$ in Figure 7. This is a resonance between the group velocity of mode 1 and the phase velocity of mode 2. The numerical speed and ratio at this point are given by, respectively, -4.937 and 0.639 in reasonable agreement with the theoretical prediction. However, this resonance is perhaps contaminated here because the resonance points $C, D, E, F$ on the dispersion curves near $G_{1,2}$ are quite close for a wide range of wavenumber $k$.
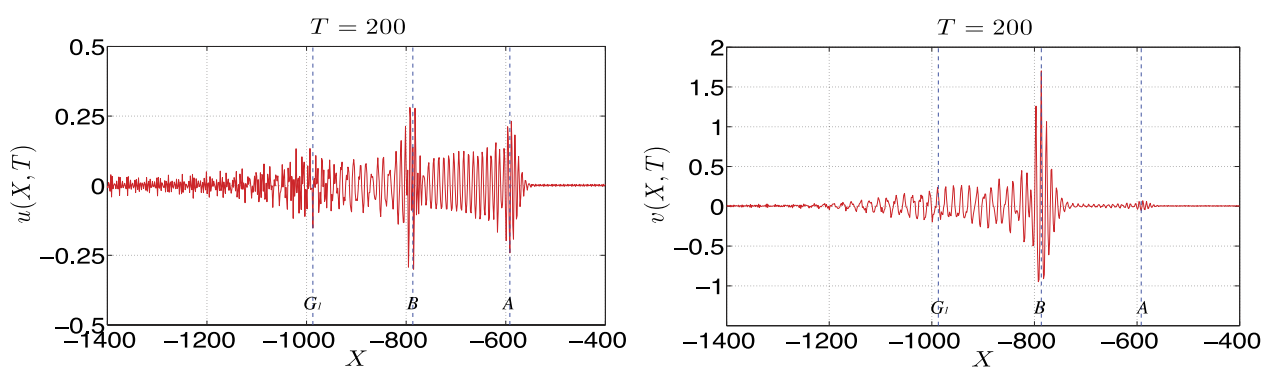

FIG. 12. Same as Figure 11 but a cross-section at $T=200$. 
TABLE I. Values of the group speed, wavenumber, and ratio, calculated using the phase speed, at each point in Figure 7.

\begin{tabular}{lccr}
\hline \hline Point & Speed & Wavenumber, $k$ & Ratio, $u_{0} / v_{0}$ \\
\hline$A$ & $-\left.2.912\right|_{\max C g_{1}}$ & 0.895 & $\left.3.692\right|_{C p_{1}}$ \\
$B$ & $-\left.3.854\right|_{\max C g_{2}}$ & 0.584 & $-\left.0.132\right|_{C p_{2}}$ \\
$C$ & $-\left.9.628\right|_{C g_{2}}$ & 1.274 & $-\left.0.602\right|_{C p_{2}}$ \\
$D$ & $-\left.12.131\right|_{C g_{2}}$ & 1.446 & $-\left.0.659\right|_{C p_{2}}$ \\
$E$ & $-\left.9.135\right|_{C g_{1}}$ & 2.251 & $\left.1.829\right|_{C p_{1}}$ \\
$F$ & $-\left.11.786\right|_{C g_{1}}$ & 2.574 & $\left.1.788\right|_{C p_{1}}$ \\
$G_{1,2}$ & $-\left.6.118\right|_{C g_{1}}$ & 1.806 & $\left.1.938\right|_{C p_{1}}$ \\
& $-\left.18.501\right|_{C g_{2}}$ & & $-\left.0.730\right|_{C p_{2}}$ \\
\hline \hline
\end{tabular}

Next, Figures 13 and 14 show the numerical results initiated using the wave packet initial conditions (128) with $k=k_{m 1}=0.895$ and ratio $r=3.692$ for mode 1 , while we set $A_{0}=0.1$. These parameters correspond to mode 1 , see point $A$ in Figure 7 . In qualitative agreement with the analogous results for a single Ostrovsky equation, we see the emergence of a nonlinear wave packet propagating to the left with speed -2.940 and ratio 3.685 , which is both close to the theoretical prediction for point $A$, see Table I. Here, we also can detect a mode 2 wave packet, corresponding to point $B$ in Figure 7, as well as some radiation due to modal energy exchange associated with the resonance point $G_{1}$. The numerically found speeds are, respectively, -4.805 for point B and -5.996 for point $G_{1}$, with ratios $R=0.411$ and $R=1.162$. In this simulation, we do not see any evidence of waves associated with the points $C, D, E, F$.

Figures 15, 16, and 17 show the numerical results commenced with wave packet initial conditions (128) with $k=k_{m 2}=0.584$ and ratio $r=-0.132$ for mode 2 . These parameters correspond to point $B$ in Figure 7. Again, we can clearly see one wave packet emerging and propagating with a speed -3.904 and ratio 0.177 , both close to the theoretical prediction for point $B$, see Table I. But here, there is also a small unsteady wave packet, seen in the $u$-component, moving with the speed -3.281 close to the theoretical prediction of $c_{g 1}=-2.912$ and ratio $R=2.555$ for a mode 1 wave packet, corresponding to point $A$ in Figure 7 and Table I. Here, we also can see the formation of wave packets to the left, corresponding to points $G_{1}$ and $C, E$ with the numerically found speeds $-6.050,-8.262$ and ratios $1.436,0.567$ also in reasonable agreement with the theoretical prediction.

Case B: A typical numerical result is shown in Figures 18 and 19 using the KdV solitary wave initial condition (126). We can clearly see a wave packet in the $u$-component identified by the vertical dashed line $A$, with speed -0.710 and ratio 4.815 . The corresponding theoretical predictions are a speed $c_{g 1}=-0.6834$ and ratio $r=33.696$, corresponding to point $A$ in Figure 8, see Table II. However, here the wave packet is strongly nonlinear, and we note that if $v$ is measured at the point where $|u|$ is a maximum, then the numerical ratio is 25 , closer to the theoretical value. Another wave packet can be clearly seen in $v$-component with speed -1.743 and ratio -0.221 . Here, the
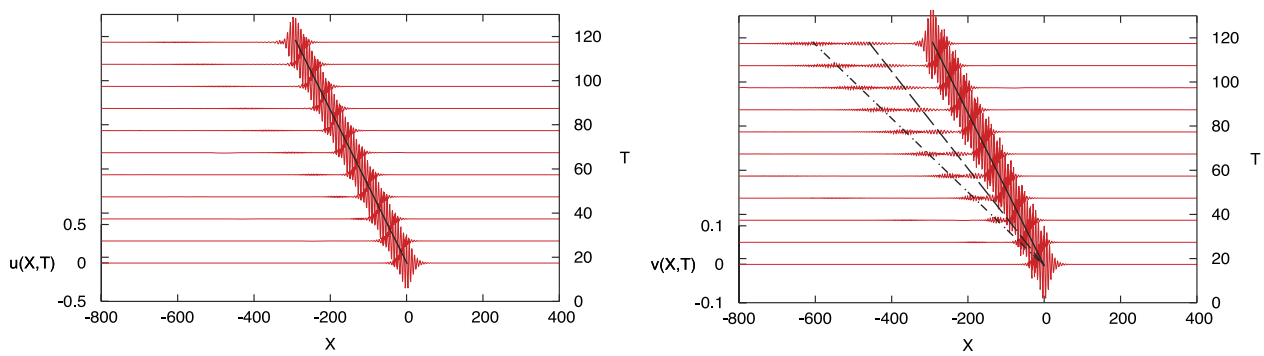

FIG. 13. Numerical simulations for Case A using the wave packet initial condition (128) with $k=k_{m 1}=0.895$ for mode 1 and $A_{0}=0.1$ and $K_{0}=0.1 \mathrm{k}$. The solid, dashed, and dashed-dotted lines refer to the points $A, B$, and $G_{1}$ in Figure 7 . 

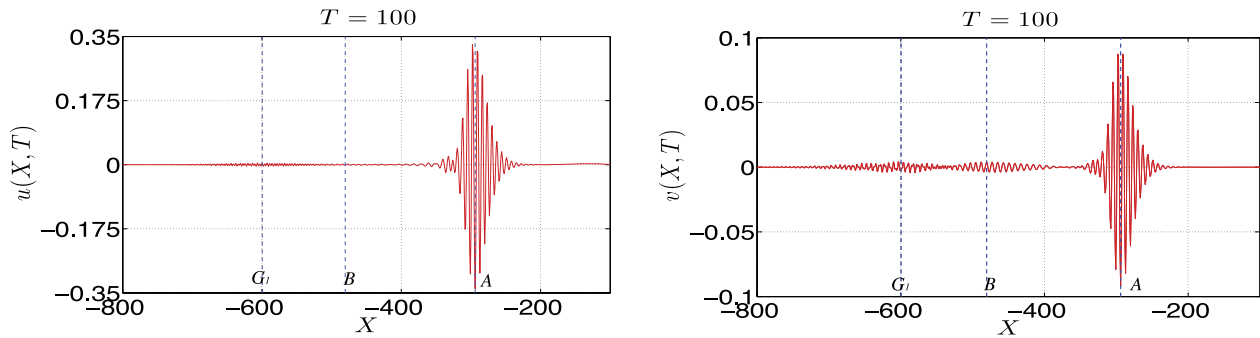

FIG. 14. Same as Figure 13 but a cross-section at $T=100$.
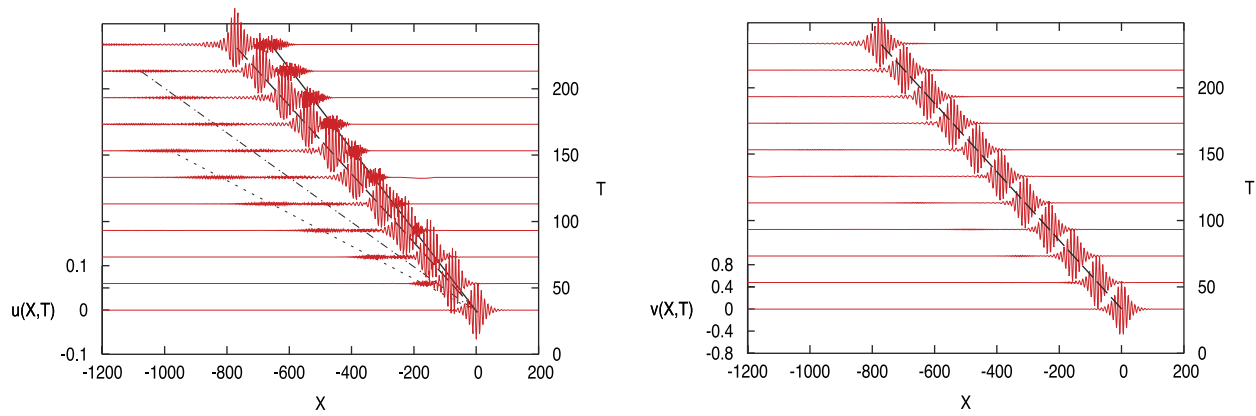

FIG. 15. Numerical simulations for Case A using the wave packet initial condition (128) with $k=k_{m 2}=0.584$ for mode 2 , and $A_{0}=0.5, K_{0}=0.1 \mathrm{k}$, and $V_{0}=1$. The solid, dashed, dashed-dotted, and dotted lines refer to the points $A, B, G_{1}$, and $(C, E)$ in Figure 7. Note that the scales for the $u$ and $v$ components are different.
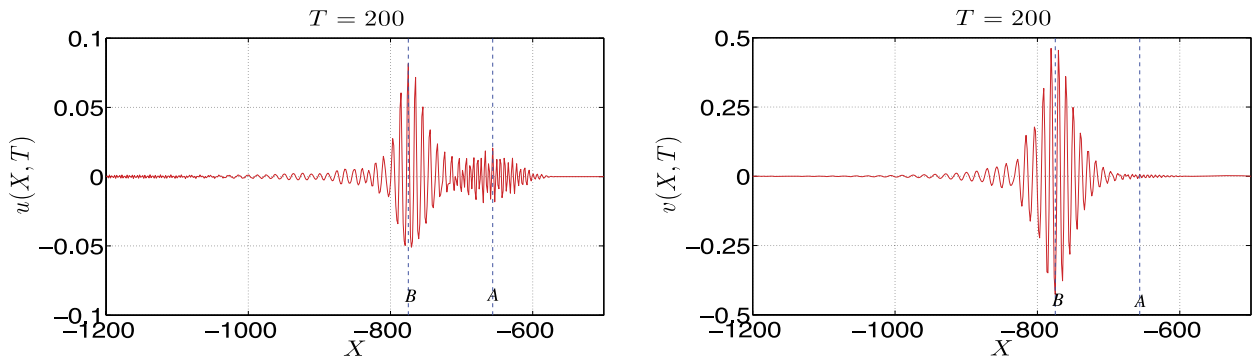

FIG. 16. Same as Figure 15 but a cross-section at $T=200$.
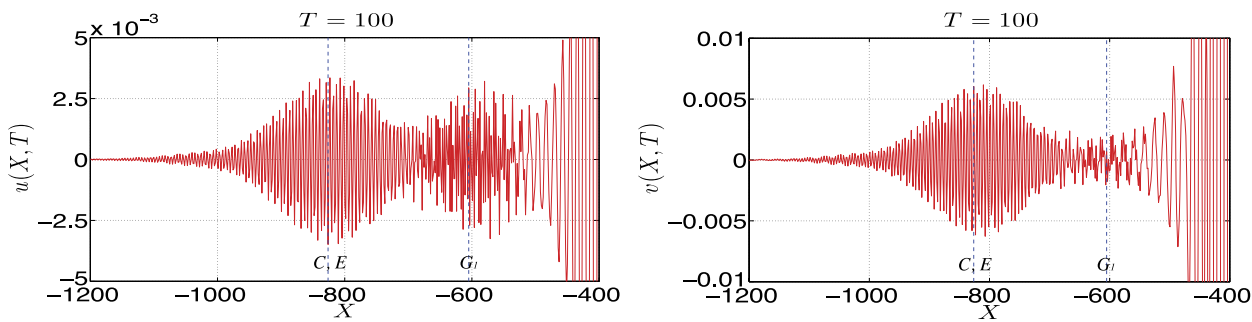

FIG. 17. Same as Figure 15 but a cross-section at $T=100$ of the domain $-1200<X<-400$ and with rescaled $u, v$. 

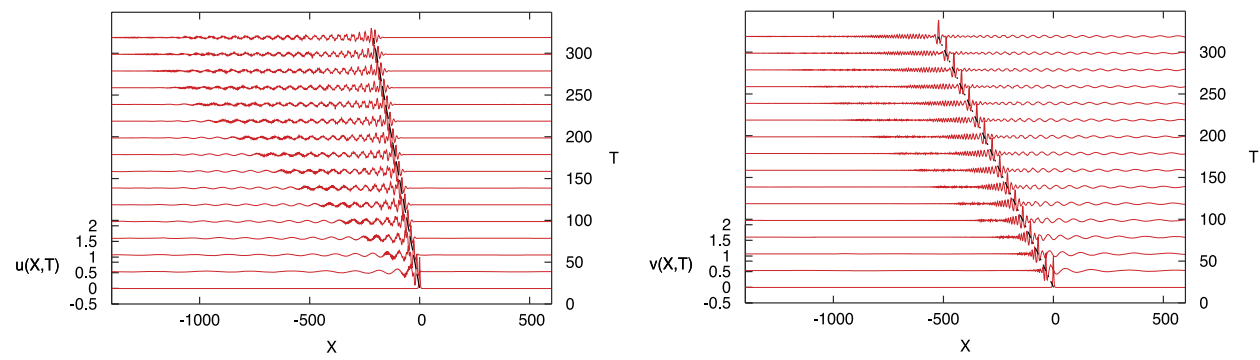

FIG. 18. Numerical simulations for Case B using a KdV initial condition of weak coupling with the parameter $a=b=1$. The dashed line in $u$-plot refers to point $A$ and the dashed-dotted line in $v$-plot refers to point $B$.

corresponding theoretical predictions are a speed -1.785 and ratio $r=-0.036$, corresponding to point $B$ in Figure 8, see Table II. Again, this wave packet is strongly nonlinear, and if $u$ is measured at the point where $|v|$ is a maximum, then the numerical ratio is -0.0229 , closer to the theoretical value. Also note that since there is considerable radiation in the plot, we cannot detect the wave packet associated to point $A$ in the $v$-plot, and similarly for the point $B$ in the $u$-plot.

In Figures 20 and 21, we use the wave packet initial condition (128), with $k=k_{m 1}=0.345$ and the ratio $R=33.696$ corresponding to a maximum group velocity $c_{g 1}=-0.683$ in mode 1 corresponding to point $A$ in Figure 8, see Table II. As expected, an unsteady wave packet emerges, clearly seen in both the $u$ and $v$ plots in the first solid line, propagating with speed -0.610 and ratio 21.261 in reasonable agreement with the theoretical predictions. The dashed line in the $v$-plot shows a wave packet propagating with speed -1.343 but the ratio cannot be measured here as in the $u$-plot, this location is the tail of the larger wave packet associated with point $A$. Based on the speed and wavenumber, we suggest this is associated with point $B$ in Figure 8, see Table II. A third small wave packet can be observed in the $v$-mode represented by the dashed-dotted line with speed -2.446 and ratio 3.160, which we associate with the resonance point $C$ for mode 1 in Figure 8 , see Table II, generated by a mode 1 unsteady wave packet associated with the point $A$. Then, a fourth small wave packet can also be observed in the $v$-mode represented by the dotted line with speed -3.057 and ratio 0.148 , which we associate with the point $E$, based on ratio and wavenumber considerations. Both these third and fourth wave packets have speeds which might be associated with the point $D_{1}$, but we have ruled out this connection due to a large disparity between the predicted and observed ratios and wavenumbers.

Figures 22 and 23 show the case when the wave packet initial condition (128) has $k=k_{s 2}=0.372$ with ratio $R=-0.036$ corresponding to a maximum phase speed in mode 2 , represented by the point $B$ in Figure 8, see Table II. In the both modes, the main feature is a steady wave packet with speed -1.787 and ratio 0.042 , see the dashed line, in good agreement with the predicted values from the dispersion relation, see Table II. There is a very small wave packet indicated by the solid line with a speed -0.461 which we associate with point $A$ based on the speed. Here, the ratio cannot be measured as this location lies in the tail of the larger wave packet associated with point $B$. There is a third wave packet shown by the blue line with speed -3.362 and ratio 0.144 , which we associate with the point $E$, based on the consideration of the speed and wavenumber, as the ratio cannot be measured accurately since in the $v$-plot, this location lies in the tail of the main wave packet. Wave packets have speeds
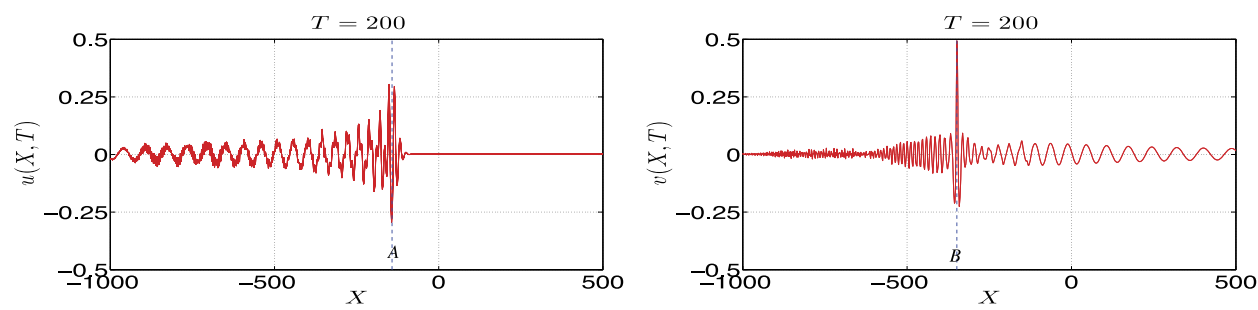

FIG. 19. Same as Figure 18 but a cross-section at $T=200$ for both modes. 
TABLE II. Values of the group speed, wavenumber, and ratio, calculated using the phase speed, at each point for Figure 8.

\begin{tabular}{llcc}
\hline \hline Point & Speed & Wavenumber, $k$ & Ratio, $u_{0} / v_{0}$ \\
\hline$A$ & $-\left.0.683\right|_{\max } C g_{1}$ & 0.345 & $\left.33.696\right|_{C p_{1}}$ \\
$B$ & $-\left.1.785\right|_{\max } C p_{2}=C g_{2}$ & 0.372 & $-\left.0.036\right|_{C p_{2}}$ \\
$C$ & $-\left.2.0430\right|_{C g_{1}}$ & 0.914 & $\left.4.012\right|_{C p_{1}}$ \\
$D_{1,2}$ & $-\left.2.987\right|_{C g_{1}}$ & 0.117 & $\left.871.768\right|_{C p_{1}}$ \\
& $-\left.0.068\right|_{C g_{2}}$ & & $-\left.0.001\right|_{C p_{2}}$ \\
$E$ & $-\left.4.676\right|_{C g_{1}}$ & 1.583 & $\left.1.779\right|_{C p_{1}}$ \\
$F_{1,2}$ & $-\left.9.722\right|_{C g_{1}}$ & 2.433 & $\left.1.287\right|_{C p_{1}}$ \\
& $-\left.27.297\right|_{C g_{2}}$ & & $-\left.0.778\right|_{C p_{2}}$ \\
\hline \hline
\end{tabular}
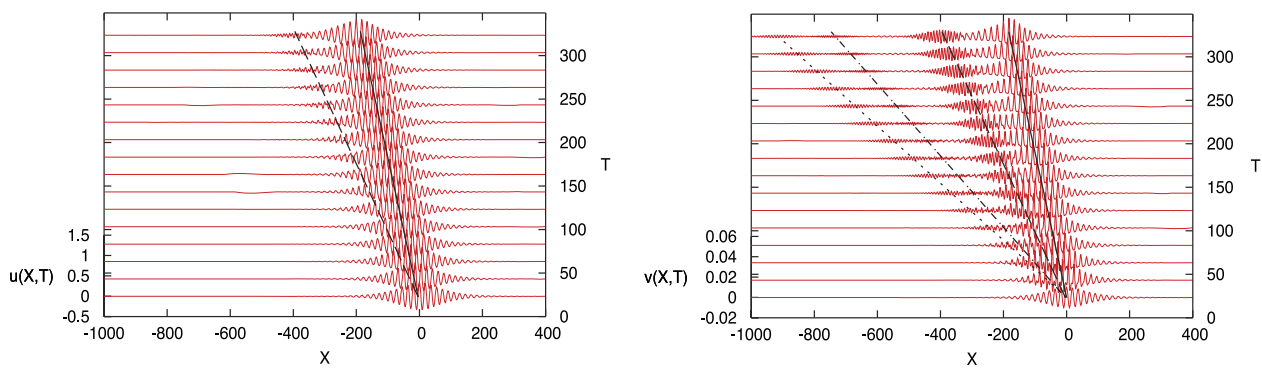

FIG. 20. Numerical simulations for Case B using a nonlinear wave packet initial condition corresponding to the value $k=k_{m 1}=0.345$ with $A_{0}=0.01, K_{0}=0.05 k$, and $V_{0}=1$. The solid, dashed, dashed-dotted, and dotted lines, respectively, refer to points $A,(B, C),\left(C, D_{1}\right)$, and $\left(D_{1}, E\right)$ in the dispersion relation.
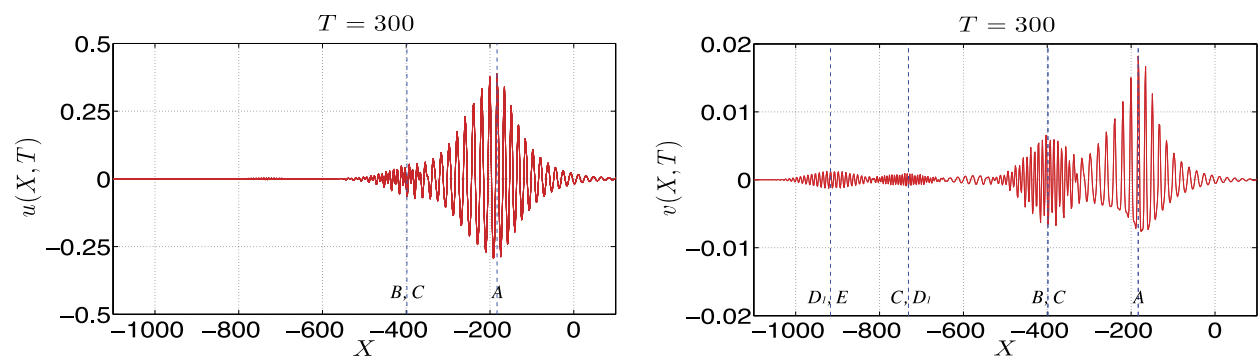

FIG. 21. Same as Figure 20 but a cross-section at $T=300$ for both modes.
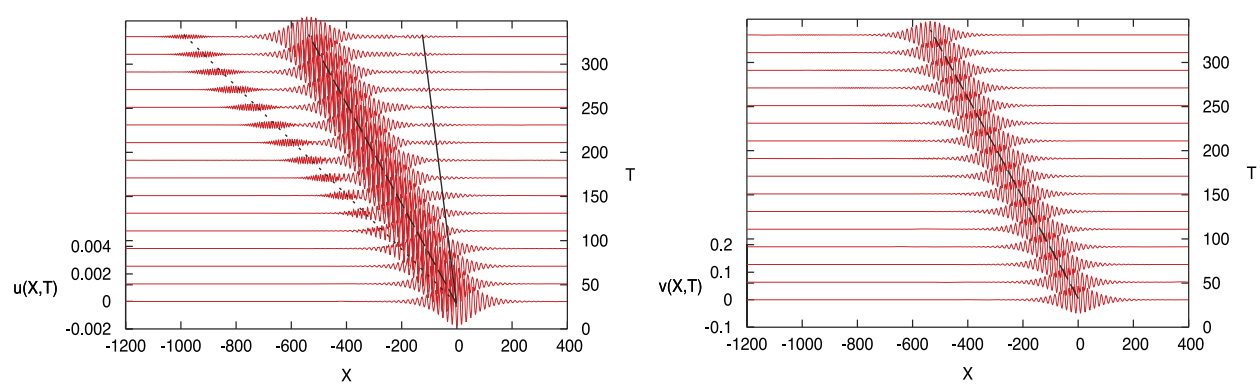

FIG. 22. Numerical simulations for Case B using a nonlinear wave packet initial condition corresponding to the value $k=k_{s 2}=0.372$ with $A_{0}=0.05, K_{0}=0.05 k$, and $V_{0}=1$. The solid, dashed, and dashed-dotted lines, respectively, refer to points $A, B$, and $\left(D_{1}, E\right)$ in the dispersion relation. 

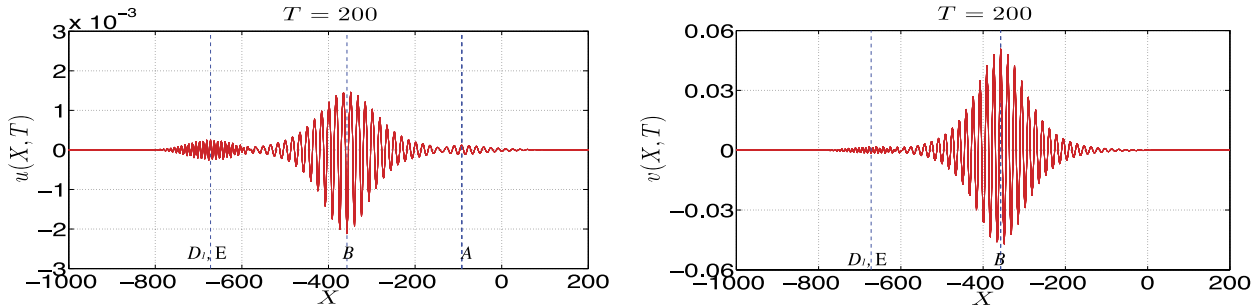

FIG. 23. Same as Figure 22 but a cross-section at $T=200$ for both modes.
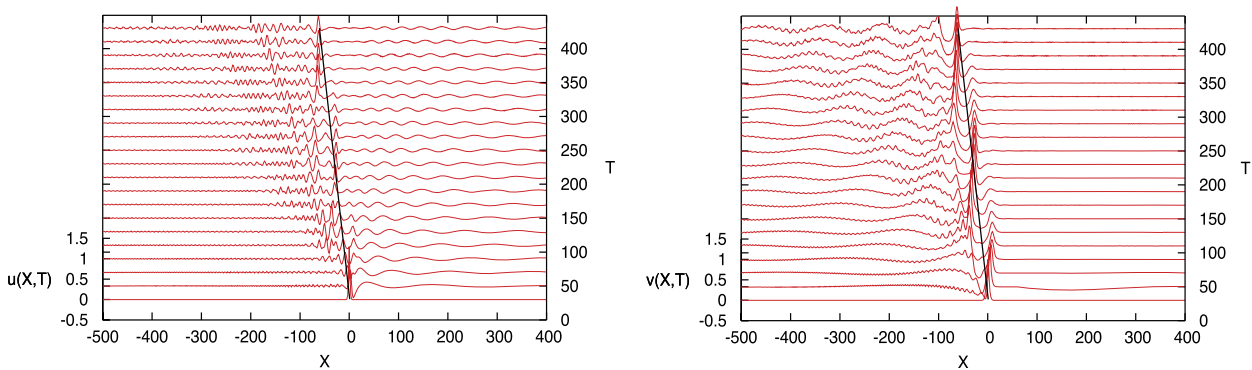

FIG. 24. Numerical simulations for Case $\mathrm{C}$ using a KdV initial condition of weak coupling (126) with $a=1.3$ and $b=1$.
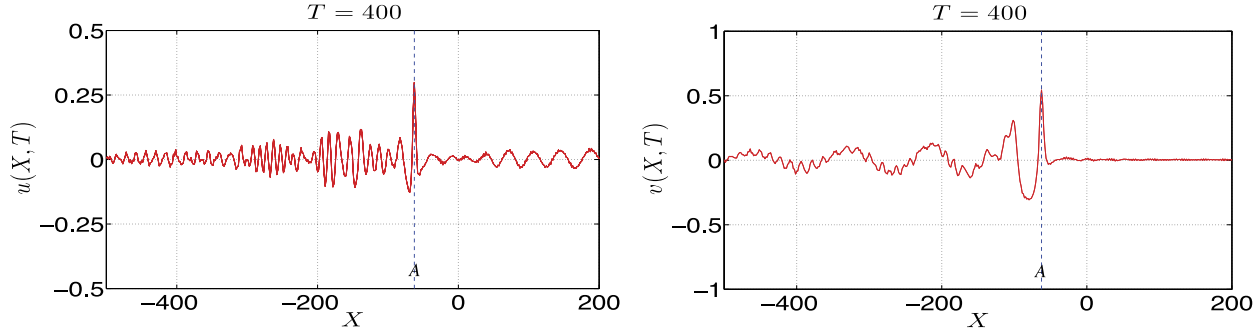

FIG. 25. Same as Figure 24 but a cross-section at $T=400$ for both modes.

TABLE III. Values of the group speed, wavenumber, and ratio, calculated using the phase speed, at each point for Figure 9.

\begin{tabular}{llcr}
\hline \hline Point & Speed, & Wavenumber, $k$ & Ratio, $u_{0} / v_{0}$ \\
\hline$A$ & $-\left.0.164\right|_{\max C g_{1}}$ & 0.306 & $\left.1.309\right|_{C p_{1}}$ \\
$B$ & $-\left.0.281\right|_{\max } C g_{1}$ & 0.152 & $\left.0.040\right|_{C p_{1}}$ \\
$C$ & $-\left.0.238\right|_{\max } C p_{2}=C g_{2}$ & 0.259 & $-\left.2.164\right|_{C p_{2}}$ \\
$D_{1,2}$ & $-\left.0.263\right|_{C g_{1}}$ & 0.245 & $\left.0.472\right|_{C p_{1}}$ \\
& $-\left.0.137\right|_{C g_{2}}$ & & $-\left.2.994\right|_{C p_{2}}$ \\
$E$ & $-\left.0.273\right|_{C g_{1}}$ & 0.404 & $\left.1.898\right|_{C p_{1}}$ \\
$F_{1,2}$ & $-\left.0.294\right|_{C g_{1}}$ & 0.199 & $\left.0.149\right|_{C p_{1}}$ \\
& $\left.0.1081\right|_{C g_{2}}$ & & $-\left.9.497\right|_{C p_{2}}$ \\
$G$ & $\left.0.075\right|_{C g_{2}}$ & 0.206 & $-\left.7.874\right|_{C p_{2}}$ \\
$H$ & $-\left.0.623\right|_{C g_{2}}$ & 0.326 & $-\left.0.932\right|_{C p_{2}}$ \\
$I$ & $-\left.0.577\right|_{C g_{1}}$ & 0.571 & $\left.1.944\right|_{C p_{1}}$ \\
$J$ & $-\left.0.722\right|_{C g_{1}}$ & 0.638 & $\left.1.914\right|_{C p_{1}}$ \\
$K$ & $-\left.0.296\right|_{\min } \boldsymbol{C g} g_{1}$ & 0.209 & $\left.0.191\right|_{C p_{1}}$ \\
$L$ & $-\left.0.681\right|_{C g_{2}}$ & 0.339 & $-\left.0.870\right|_{C p_{2}}$ \\
$M$ & $-\left.0.770\right|_{C g_{1}}$ & 0.659 & $\left.1.904\right|_{C p_{1}}$ \\
$N$ & $\left.0.111\right|_{C g_{2}}$ & 0.199 & $-\left.9.651\right|_{C p_{2}}$ \\
\hline \hline
\end{tabular}



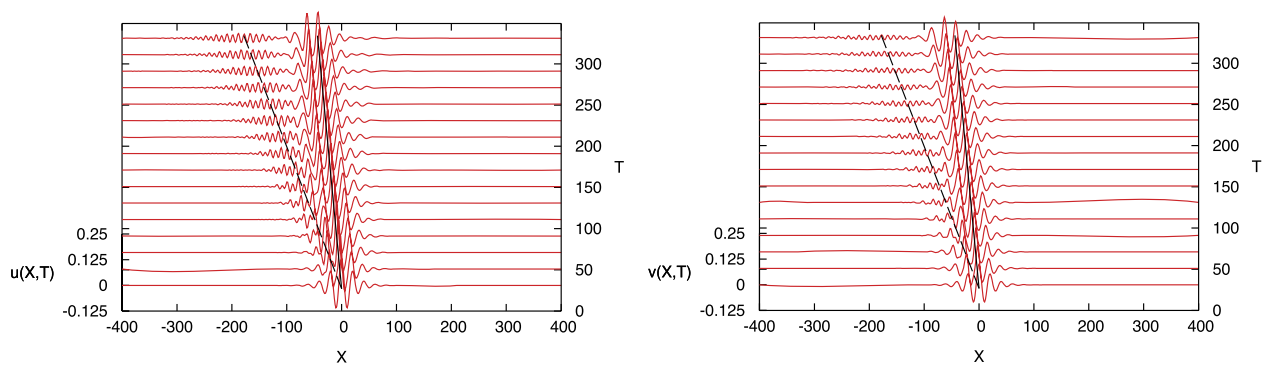

FIG. 26. Numerical simulations for Case $\mathrm{C}$ using the wave packet initial condition (128) with $k=k_{m 1}=0.306$ corresponding to point $A$ with $A_{0}=0.1, K_{0}=0.2 k$, and $V_{0}=1$. The solid and dashed lines, respectively, refer to points $A$ and $(I, J, M)$ indicated in Figure 27.

which might be associated with the point $D_{1}$, but we have ruled out this connection due to a large disparity between the predicted and observed ratio and wavenumber.

Case C: Case C is analogous to Case B. A typical numerical result is shown in Figures 24 and 25 using the KdV solitary wave initial condition (126). But here, we chose $\gamma_{1} \neq \gamma_{2}$ in order that the ratio $a / b$ should coincide with the predicted ratio 1.3 corresponding to the point $A$ in Figure 9 . A strongly nonlinear unsteady wave packet emerges, denoted by the vertical line $A$ in Figure 25, with speed -0.156 and ratio 0.5496 , in agreement for the speed with the theoretical predictions from the point $A$ in the dispersion plots of Figure 9 and Table III. This wave packet has a phase speed which is very close to the group velocity over the range of wavenumbers from the point $D$ to $E$, leading to strongly nonlinear effects and difficulty in numerically determining a ratio. In Figures 24 and 25 , there is also evidence of significant radiation both to the right and to the left of the main wave packet. The waves to the right with positive speed can be associated with the points $F_{2}$ and/or $N$ as these have a positive group velocity for mode 2 and a ratio of nearly -10 , which mean that the amplitude in the $v$-plot is too small to be seen. Although the points $F$ and $N$ are very close, they have a different interpretation. The point $F_{2}$ is a resonance between $c_{g 1}$ and $c_{p 2}$, while the point $N$ is a resonance between the speed at the minimum point of $c_{g 1}$ with $c_{p 2}$. Moreover, this wave to the right has the appearance of a linear dispersive wave, and hence there is no very clear identifiable speed or wavenumber. The waves to the left show both small-scale and large scale features in both $u$ and $v$, with the small-scale features more prominent in $u$ and the large-scale features more prominent in $v$. The large-scale feature may be associated with either $B$ or $K$ and the small-scale with either $J$ or $M$. That is, these are mode 1 waves associated with turning points in the group velocity and a resonance with the phase velocity. Also note that for both $B$ and $K$, the ratio is such that $v$ dominates, while for $J$ and $M$, it is $u$ that dominates, features consistent with the numerical simulation. Thus, overall all features in the numerical simulation can be associated with the turning points in the group velocity curve $c_{g 1}$ for mode 1 .

As noted above, the group velocity curve $c_{g 1}$ for mode 1 has three turning points, while there are no such turning points for $c_{g 2}$. To examine each of these, we first examined the turning point $A$ in Figure 9 and Table III and used the wave packet initial condition (128) with wavenumber $k=k_{m 1}=0.306$ and ratio $R=1.309$. The numerical results are shown in Figures 26 and 27 and the
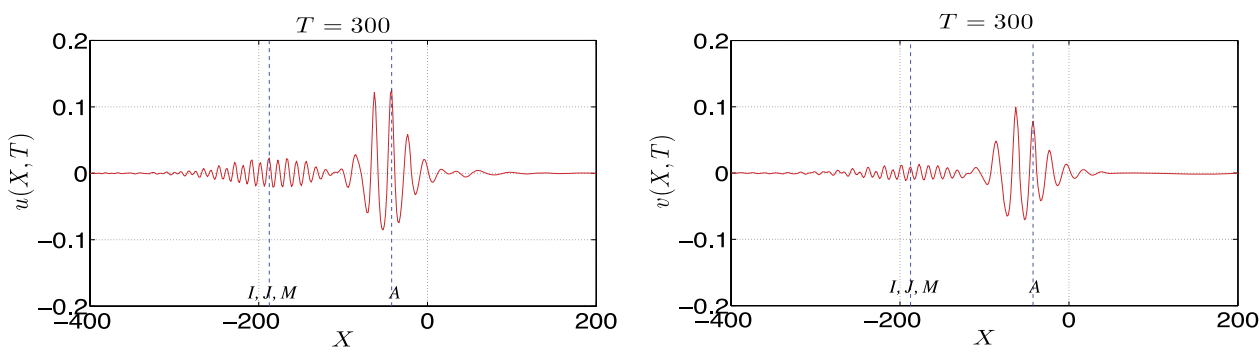

FIG. 27. Same as Figure 26 but a cross-section at $T=300$ for both modes. 

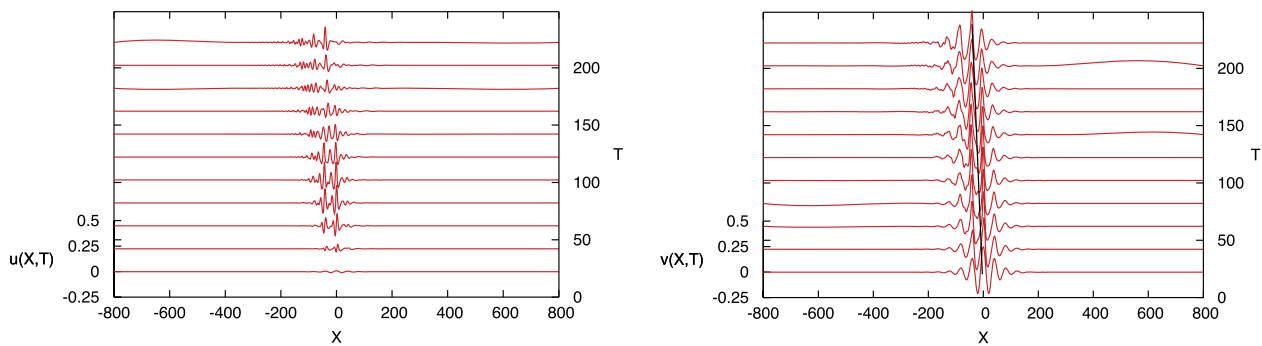

FIG. 28. Numerical simulations for Case $\mathrm{C}$ using the wave packet initial condition (128) with $k=0.152$ corresponding to point $B$ with $A_{0}=0.25, K_{0}=0.2 k$, and $V_{0}=1$.
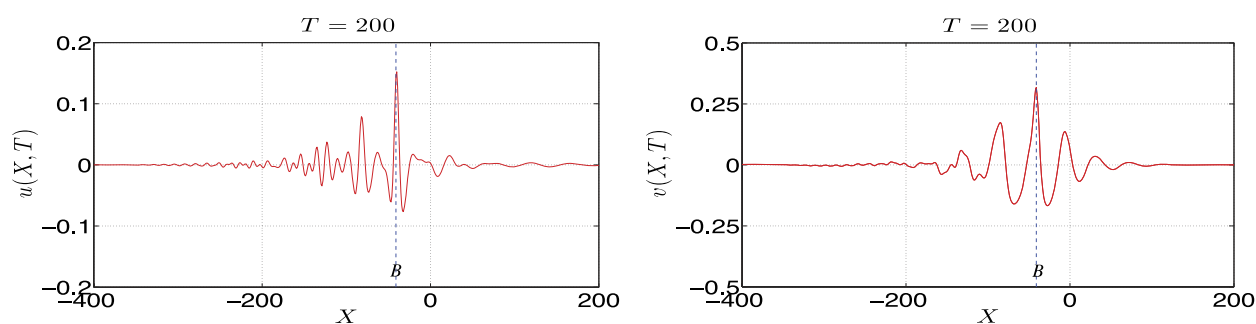

FIG. 29. Same as Figure 28 but a cross-section at $T=200$ for both modes.
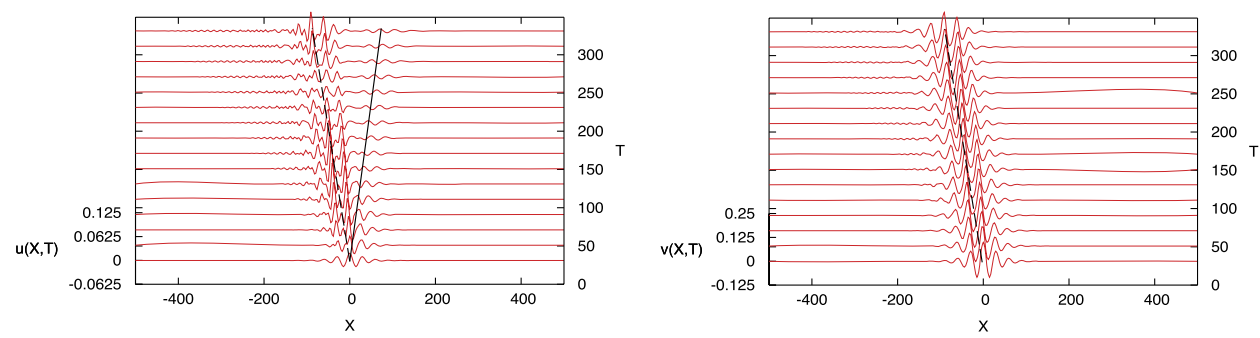

FIG. 30. Numerical simulations for Case $\mathrm{C}$ using the wave packet initial condition (128) with $k=0.209$ corresponding to point $K$ with $A_{0}=0.1, K_{0}=0.2 k$, and $V_{0}=1$. The solid and dashed lines, respectively, refer to points $\left(F_{2}, G, N\right)$ and $\left(D_{1}, F_{1}, K\right)$ in Figure 9.
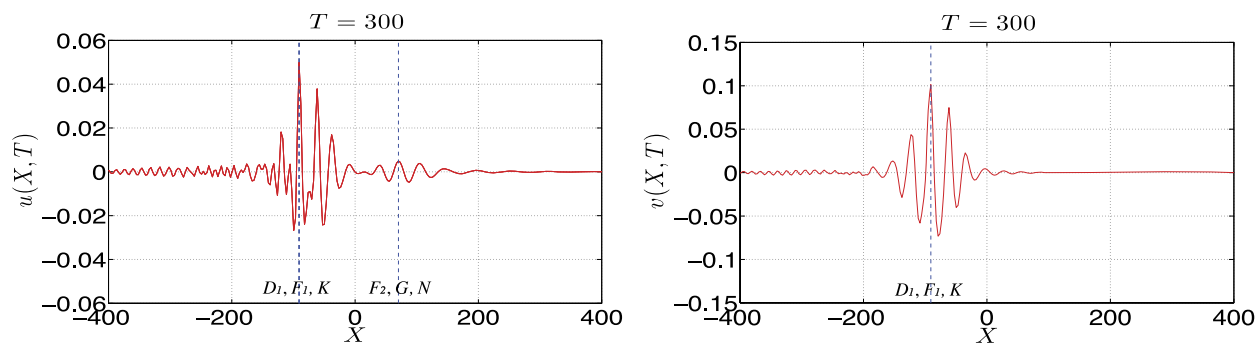

FIG. 31. Same as Figure 30 but a cross-section at $T=300$ for both modes.
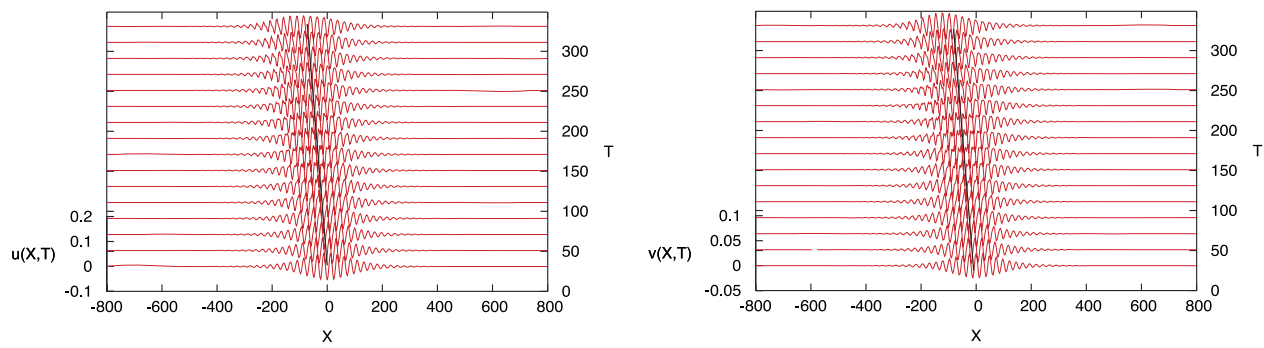

FIG. 32. Numerical simulations for Case $\mathrm{C}$ using the wave packet initial condition (128) with $k=k_{s 2}=0.259$ corresponding to point $C$ with $A_{0}=0.025, K_{0}=0.05 k$, and $V_{0}=1$. 

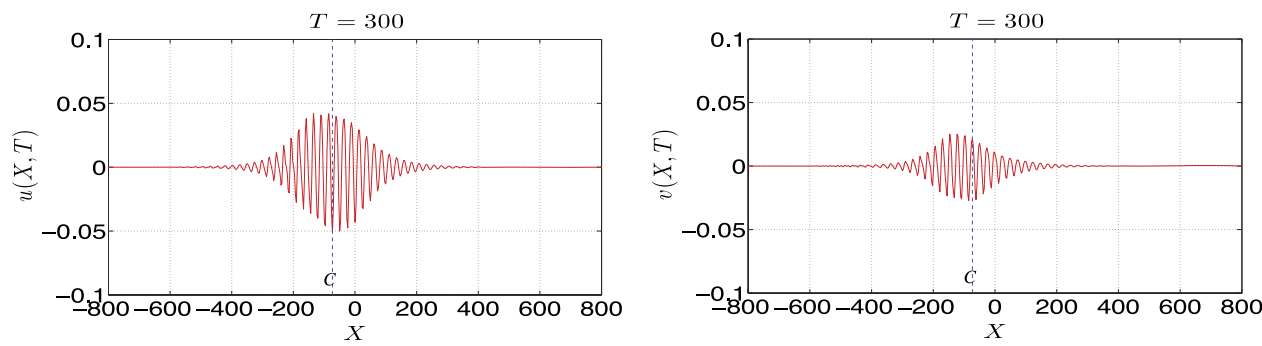

FIG. 33. Same as Figure 32 but a cross-section at $T=300$ for both modes.

emergence of a nonlinear wave packet is clearly seen. At the vertical line $A$, the speed is -0.146 with ratio 1.387 , in agreement with the theoretical prediction. There is a secondary wave packet now discernible on the vertical line $I$, moving with speed -0.625 and ratio 1.910 , which from the dispersion relation in Figure 9 is identified with the point $I$, which is a resonance between the maximum value of the phase speed of mode 2 (point $C$ ) with mode 1 . However, we note that the resonance points $J, M$ are close by with similar values and so may also be relevant.

Next, we used the wave packet initial condition associated with the turning point $B$ in Figure 9, with $k=0.152$ and $A_{0}=0.25, K_{0}=0.2 k$, and $V_{0}=1$. The numerical result is shown in Figures 28 and 29. A nonlinear wave packet emerges with speed -0.205 and ratio 0.460 , whereas the predicted values are -0.281 and 0.04 in Table III. The speed is approximately consistent with the theoretical prediction for point $B$ but the ratio is not. However, we note here that due to the variability in the emerging wave packets in the $u$-variable, the ratio is quite hard to determine here. This may be due to contamination with waves associated with the points $A$ or $D_{2}$.

The corresponding numerical result for an initial condition associated with the turning point $K$ are shown in Figures 30 and 31. A strongly nonlinear wave packet emerges, with speed -0.303 and ratio 0.492 , can be seen in both the $u$ and $v$ plots, and is in reasonable agreement with the theoretical prediction. However, the resonance points $D_{1}, F_{1}$ have similar speeds, and the strong nonlinearity suggests there may be some interaction here leading to difficulty in determining a numeral ratio. There is also a small wave propagating to the right, seen in the $u$-plot, with the speed 0.234 and the ratio 4.939, indicated by the vertical line $N$, which can be associated with one or more of the resonance points $N, F_{2}, G$ in Figure 9.

Finally, we turn to the simulation associated with the turning point $C$ in Figure 9 using the wave packet initial condition (128) with $A_{0}=0.025, K_{0}=0.05 k$, and $V_{0}=1$. The numerical result is shown in Figures 32 and 33. In this case, a steady wave packet clearly emerges, indicated by the solid line, with speed -0.244 and ratio 1.874 , in good agreement with the predicted theoretical values. Note that the resonance point $E$ has a similar speed, but quite different wavenumber, and indeed we do not see that wave forms associated with this point.

Case D: A typical numerical result is shown in Figures 34 and 35 using the KdV solitary wave initial condition (126). The numerical results show two steady wave packets emerging, as expected, with speeds $-0.146,-0.586$ and ratios $10.136,1.753$ associated with the vertical lines $A$ and $B$, respectively, in Figure 35, in reasonable agreement with the theoretical values. These wave packets
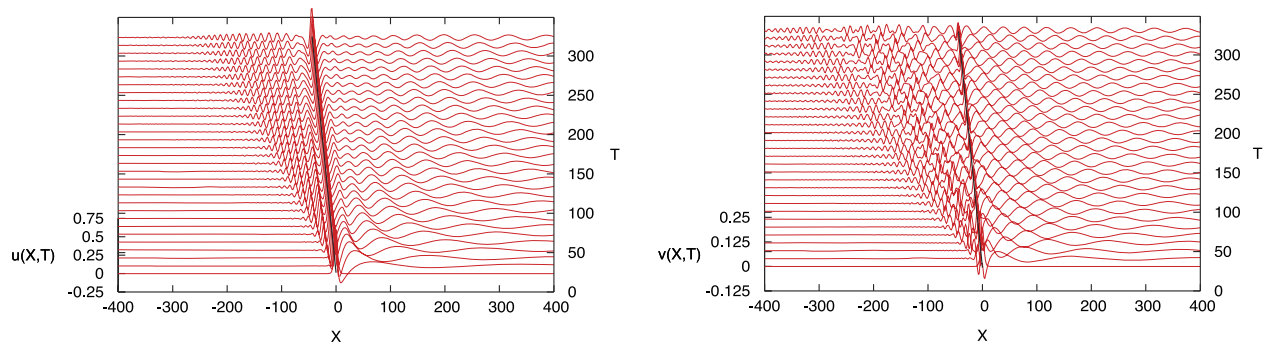

FIG. 34. Numerical simulations for Case D using a KdV initial condition of weak coupling (126) with $a=0.6$ and $b=0.2$. The solid line in both plots refers to point $A$. 

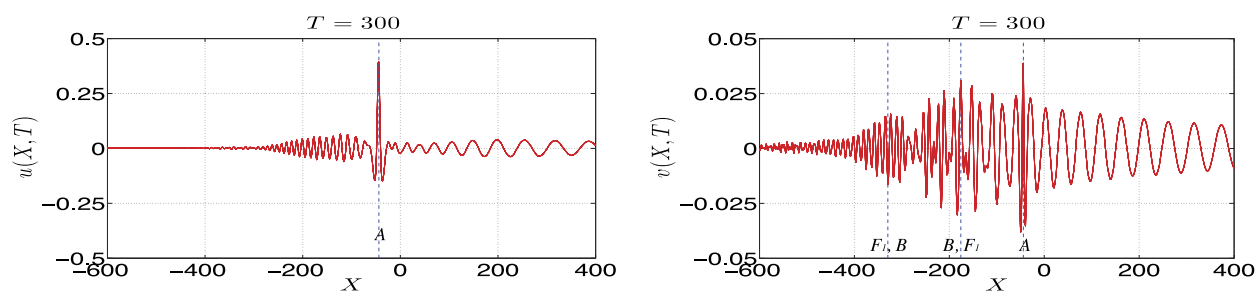

FIG. 35. Same as Figure 34 but a cross-section at $T=300$ for both modes.

are strongly nonlinear and there is considerable evidence of resonances and radiation. In particular, the vertical line $F$ in Figure 35 is interpreted as an interaction between the points $B$ and $F_{1}$, the latter being a resonance between the group velocity of mode 1 and phase speed of mode 2 , see Figure 10 and Table IV. There is also a transient wave propagating to the right probably due to fact that the negative signs of both $\beta$ and $\mu$ allow both modes to have positive group velocities for low wavenumbers.

There are two different wavenumbers to consider when we use the wave packet initial condition (128) corresponding to the points $A$ and $B$ in Figure 10. First, we choose $k=k_{s 1}=0.3221$ and $R=10.9729$ corresponding to the point $A$ in Figure 10, see Table IV. The numerical results are shown in Figures 36 and 37 and we see that the solution is dominated by a steady mode 1 wave packet, with speed -0.189 and ratio 7.934 in good agreement with the theoretical values. Another wave packet can be seen corresponding to the points $B, F_{2}$ in Figure 10, with speed -0.846 and ratio 3.056. Here, there is some interaction between these two points. Further, there is a very small wave packet associated with the points $F_{2}$ in Figure 10, with speed -1.706 and ratio 0.368 , in good agreement to theoretical values; although there may be some contamination here due to the point $D$, which has a similar speed.

Second, we use the wave packet initial condition (128) with $k=k_{s 2}=0.395$ and ratio, $R$ $=-0.105$ corresponding to the point $B$ in Figure 10, see Table IV. The numerical results are shown in Figures 38 and 39 and the solution is now dominated by a steady mode 2 wave packet, as expected, with speed -0.820 and ratio -0.229 , in good agreement with the theoretical values. There is also some interaction with the point $F_{1}$ here, seen in the $u$-plot where two wavenumbers can be seen. However, the dispersion curves in Figure 10 show that here there are potential resonances with mode 1 at $k=0.1168$ and $k=1.0657$, associated with the points $C$ and $D$, see Table IV. There is no discernible evidence here of radiation into the wavenumber $k=0.1168$ due to the large ratio of $O(200)$ needed, but a wave packet is seen with wavenumber $k=1.0657$, indicated by blue vertical line $D$ in Figure 39, with the speed -2.014 and ratio 0.814 , in reasonable agreement with the theoretical prediction; although there could also be some interaction with the point $F_{2}$ here, which has quite similar values. Another small wave packet can be seen, possibly corresponding to point $G_{1}$ in Figure 39 with the speed -2.578 and ratio 0.503 .

TABLE IV. Values of the group speed, wavenumber, and ratio, calculated using the phase speed, at each point for Figure 10.

\begin{tabular}{lccr}
\hline \hline Point & Speed & Wavenumber, $k$ & Ratio, $u_{0} / v_{0}$ \\
\hline$A$ & $-\left.0.197\right|_{\max } C p_{1}=C g_{1}$ & 0.322 & $\left.10.973\right|_{C p_{1}}$ \\
$B$ & $-\left.0.747\right|_{\max } C p_{2}=C g 2$ & 0.395 & $-\left.0.105\right|_{C p_{2}}$ \\
$C$ & $\left.0.692\right|_{C g_{1}}$ & 0.117 & $\left.180.200\right|_{C p_{1}}$ \\
$D$ & $-\left.1.781\right|_{C g_{1}}$ & 1.066 & $\left.1.011\right|_{C p_{1}}$ \\
$E_{1,2}$ & $\left.0.033\right|_{C g_{1}}$ & 0.231 & $\left.25.339\right|_{C p_{1}}$ \\
& $-\left.0.241\right|_{C g_{2}}$ & & $-\left.0.028\right|_{C p_{2}}$ \\
$F_{1,2}$ & $-\left.0.931\right|_{C g_{1}}$ & 0.664 & $\left.2.117\right|_{C p_{1}}$ \\
& $-\left.1.761\right|_{C g_{2}}$ & & $-\left.0.334\right|_{C p_{2}}$ \\
$G_{1,2}$ & $-\left.2.894\right|_{C g_{1}}$ & 1.438 & $\left.0.794\right|_{C p_{1}}$ \\
& $-\left.8.182\right|_{C g_{2}}$ & & $-\left.0.892\right|_{C p_{2}}$ \\
\hline \hline
\end{tabular}



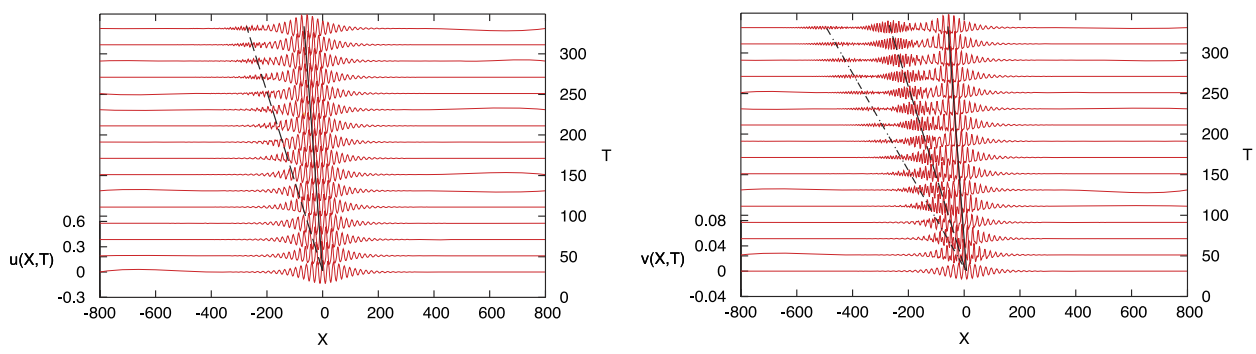

FIG. 36. Numerical simulations for Case D using the wave packet initial condition (128) with $k=k_{m 1}=0.322$ corresponding to the point $A$ in Figure 10, with $A_{0}=0.05, K_{0}=0.05 k$, and $V_{0}=0.25$. The solid, dashed, and dashed-dotted lines, respectively, refer to points $A,\left(B, F_{1}\right)$, and $\left(F_{2}, D\right)$.
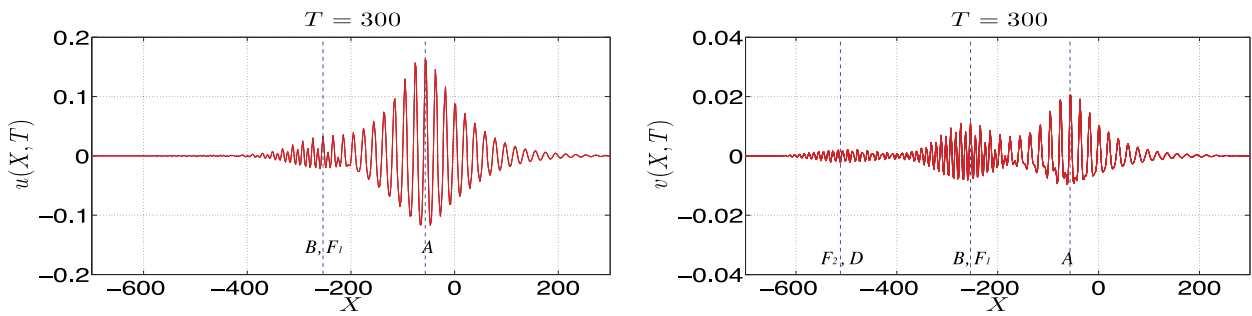

FIG. 37. Same as Figure 36 but a cross-section at $T=300$ for both modes.
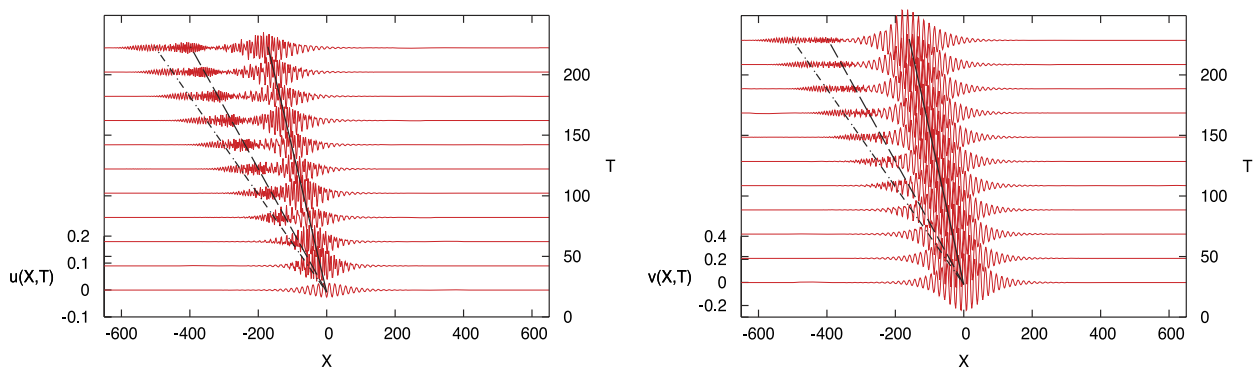

FIG. 38. Numerical simulations for Case D using the wave packet initial condition (128) with $k=k_{s 2}=0.395$, corresponding to the point $B$ in Figure 10 with $A_{0}=0.25, K_{0}=0.05 k$, and $V_{0}=1$. The solid, dashed, and dashed-dotted lines, respectively, refer to points $\left(B, F_{1}\right),\left(F_{2}, D\right)$, and $G_{1}$.
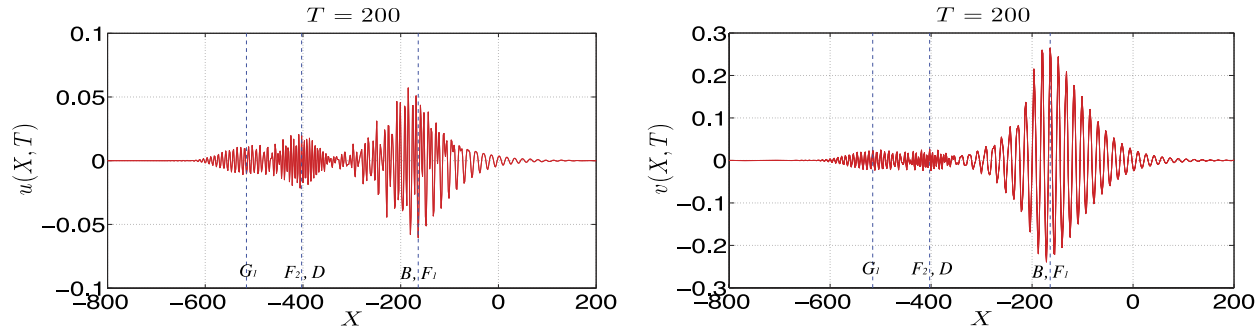

FIG. 39. Same as Figure 38 but a cross-section at $T=200$ for both modes. 


\section{SUMMARY AND DISCUSSION}

In this paper, we have briefly reviewed the derivation of coupled Ostrovsky equations for resonantly interacting weakly nonlinear long oceanic internal waves, presented in detail in our previous work. ${ }^{19}$ The resulting system (42) and (43) describes the evolution of the amplitudes of two linear long wave modes whose linear long wave phase speeds are nearly coincident. In an extension of our previous work, here we focus on the effect of a background shear flow, using a three-layer model as a guide to the possible values that the normalised coefficients may take. The significant difference that emerges is that the coefficients $\beta, \mu$ of the rotation terms in the coupled Ostrovsky equations (49) and (50), are not necessarily equal, or indeed positive, as is the case in the absence of a background shear flow. Instead, there are four essentially different cases corresponding to different sign combinations of $\beta$ and $\mu$.

Then, the system was examined numerically, using two different initial conditions. First, the initial condition was a solitary wave type, based on an approximation to the coupled KdV systems obtained when the rotation terms are removed, and for which there is no a priori wavenumber selection. Second, the initial condition was a wave packet based on certain predicted wavenumbers, obtained from the linear dispersion relation where either the phase velocity or the group velocity, has a turning point. The former can be associated with the possible emergence of a nonlinear steady wave packet and the latter with the possible emergence of an unsteady nonlinear wave packet. These two contrasting scenarios were examined numerically for each of the four cases. In each case, we can identify these predicted wave packets as the dominant feature of the numerical solution. However, in many cases, there was also evidence of nonlinear interactions generating other wave packets associated with some of the possible resonant points identified on each linear dispersion curve. Thus, in comparison with the simulations of the single Ostrovsky equation reported by Grimshaw and Helfrich ${ }^{11}$ where only a single unsteady nonlinear wave packet typically emerges, the coupled system (42) and (43) can support a wide variety of nonlinear wave packets. Importantly, we have shown that the dominant features of the observed dynamical behaviours can be classified and interpreted in terms of the main features of the relevant dispersion curves. This is a first step towards predicting the long-time asymptotic behaviour of solutions of the initial-value problems for this coupled system of equations.

Although we have used a particular three-layer model to illustrate the range of possible scenarios, based, in particular, on the signs of the rotational coefficients $\beta, \mu$, we suggest that similar combinations of stratification and current shear will lead to the same range of possible sign combinations, and hence to the same range of complex dynamical behaviour. Thus, we expect that these kinds of nonlinear wave packets may be found under certain oceanic conditions, and could be possibly observed in laboratory experiments, similar to that of Grimshaw et al. ${ }^{23}$ for the generation of the unsteady wave packets described by the single Ostrovsky equation. Of course, in reality in the ocean the wave packets found here may be affected by dissipation and the competing effects of topography as the waves shoal shoreward, see Grimshaw et al. ${ }^{24}$ Nevertheless, they can provide a useful framework for the interpretation of the observed wave phenomena.

\section{ACKNOWLEDGMENTS}

One of the authors, A. Alias, is supported by Universiti Malaysia Terengganu and the Ministry of Higher Education of Malaysia.

${ }^{1}$ R. H. J. Grimshaw, “Internal solitary waves,” in Environmental Stratified Flows, edited by R. Grimshaw (Kluwer, Boston, 2001), pp. 1-27.

${ }^{2}$ R. H. J. Grimshaw, L. A. Ostrovsky, V. I. Shrira, and Yu. A. Stepanyants, "Long nonlinear surface and internal gravity waves in a rotating ocean," Surv. Geophys. 19(4), 289-338 (1998).

${ }^{3}$ K. R. Helfrich and W. K. Melville, "Long nonlinear internal waves," Annu. Rev. Fluid Mech. 38, 395-425 (2006).

${ }^{4}$ L. A. Ostrovsky, "Nonlinear internal waves in a rotating ocean," Oceanology 18(2), 119-125 (1978).

${ }^{5}$ A. I. Leonov, "The effect of the Earth's rotation on the propagation of weak nonlinear surface and internal long oceanic waves," Ann. N. Y. Acad. Sci. 373(1), 150-159 (1981).

${ }^{6}$ K. R. Helfrich, "Decay and return of internal solitary waves with rotation," Phys. Fluids 19(2), 026601 (2007)

${ }^{7}$ R. H. J. Grimshaw, "Evolution equations for weakly nonlinear, long internal waves in a rotating fluid," Stud. Appl. Math. 73, 1-33 (1985) 
${ }^{8}$ R. H. J. Grimshaw, "Models for nonlinear long internal waves in a rotating fluid," Fundam. Appl. Hydrophys. 6, 4-13 (2013).

${ }^{9}$ R. H. J. Grimshaw and K. Helfrich, "The effect of rotation on internal solitary waves," IMA J. Appl. Math. 77(3), 326-339 (2012).

${ }^{10}$ R. H. J. Grimshaw, J.-M. He, and L. A. Ostrovsky, "Terminal damping of a solitary wave due to radiation in rotational systems," Stud. Appl. Math. 101(2), 197-210 (1998).

${ }^{11}$ R. H. J. Grimshaw and K. Helfrich, "Long-time solutions of the Ostrovsky equation,” Stud. Appl. Math. 121(1), 71-88 (2008).

${ }^{12}$ D. Yagi and T. Kawahara, "Strongly nonlinear envelope soliton in a lattice model for periodic structure," Wave Motion 34(1), 97-107 (2001).

13 T. Gerkema, "A unified model for the generation and fission of internal tides in a rotating ocean," J. Mar. Res. 54(3), 421-450 (1996).

14 V. N. Galkin and Yu. A. Stepanyants, "On the existence of stationary solitary waves in a rotating fluid," J. Appl. Math. Mech. 55(6), 939-943 (1991).

${ }^{15}$ M. A. Obregon and Yu. A. Stepanyants, "Oblique magneto-acoustic solitons in a rotating plasma," Phys. Lett. A 249(4), 315-323 (1998)

${ }^{16}$ C. Eckart, "Internal waves in the ocean," Phys. Fluids 4, 791-799 (1961).

${ }^{17}$ J. A. Gear and R. H. J. Grimshaw, “Weak and strong interactions between internal solitary waves,” Stud. Appl. Math. 70(1), 235-258 (1984)

${ }^{18}$ R. H. J. Grimshaw, "Coupled Korteweg-de Vries equations," in Without Bounds: A Scientific Canvas of Nonlinearity and Complex Dynamics, edited by R. G. Rubio, Y. S. Ryazantsev, V. M. Starov, G.-X. Huang, A. P. Chetverikov, P. Arena, A. A. Nepomnyashchy, A. Ferrus, and E. G. Morozov (Springer, Berlin Heidelberg, 2013), pp. 317-333.

${ }^{19}$ A. Alias, R. H. J Grimshaw, and K. R. Khusnutdinova, "On strongly interacting internal waves in a rotating ocean and coupled Ostrovsky equations," Chaos 23(2), 023121 (2013).

${ }^{20}$ K. R. Khusnutdinova, A. M. Samsonov, and A. S. Zakharov, "Nonlinear layered lattice model and generalized solitary waves in imperfectly bonded structures," Phys. Rev. E: Stat., Nonlinear, Soft Matter Phys. 79, 056606 (2009).

${ }^{21}$ K. R. Khusnutdinova and K. M. Moore, "Initial-value problem for coupled Boussinesq equations and a hierarchy of Ostrovsky equations," Wave Motion 48(8), 738-752 (2011).

${ }^{22}$ R. H. J. Grimshaw and G. Iooss, "Solitary waves of a coupled Korteweg-de Vries system," Math. Comput. Simul. 62(12), 31-40 (2003).

${ }^{23}$ R. H. J. Grimshaw, K. R. Helfrich, and E. R. Johnson, "Experimental study of the effect of rotation on nonlinear internal waves," Phys. Fluids 25(5), 056602 (2013).

${ }^{24}$ R. Grimshaw, C. Guo, K. Helfrich, and V. Vlasenko, "Combined effect of rotation and topography on shoaling oceanic internal solitary waves," J. Phys. Oceanol. 44, 1116-1132 (2014). 